\title{
Using radio triangulation to understand the origin of two subsequent type II radio bursts
}

\author{
I. C. Jebaraj ${ }^{1,2}$, J. Magdalenić ${ }^{1}$, T. Podladchikova ${ }^{3}$, C. Scolini ${ }^{2,1}$, J. Pomoell ${ }^{4}$, A. M. Veronig ${ }^{5,6}$, K. Dissauer ${ }^{5}$, \\ V. $\operatorname{Krupar}^{7,8}$, E. K. J. Kilpua ${ }^{4}$, and S. Poedts ${ }^{2,9}$
}

1 SIDC, Royal Observatory of Belgium, Brussels, Belgium e-mail: immanuel.jebaraj@oma.be

2 Centre for mathematical Plasma Astrophysics, KU Leuven, Leuven, Belgium

3 Skolkovo Institute of Science and Technology, Moscow, Russia

${ }^{4}$ University of Helsinki, Helsinki, Finland

5 Institute of Physics, University of Graz, Graz, Austria

${ }^{6}$ Kanzelhöhe Observatory for Solar and Environmental Research, University of Graz, Graz, Austria

7 Goddard Planetary Heliophysics Institute, University of Maryland, Baltimore County, Baltimore, MD 21250, USA

8 Heliospheric Physics Laboratory, Heliophysics Division, NASA Goddard Space Flight Center, Greenbelt, MD 20771, USA

9 Institute of Physics, University of Maria Curie-Skłodowska, 20-031 Lublin, Poland

Received 6 December 2019 / Accepted 7 May 2020

\begin{abstract}
Context. Eruptive events such as coronal mass ejections (CMEs) and flares accelerate particles and generate shock waves which can arrive at Earth and can disturb the magnetosphere. Understanding the association between CMEs and CME-driven shocks is therefore highly important for space weather studies.

Aims. We present a study of the CME/flare event associated with two type II bursts observed on September 27, 2012. The aim of the study is to understand the relationship between the observed CME and the two distinct shock wave signatures.

Methods. The multiwavelength study of the eruptive event (CME/flare) was complemented with radio triangulation of the associated radio emission and modelling of the CME and the shock wave employing MHD simulations.

Results. We found that, although temporal association between the type II bursts and the CME is good, the low-frequency type II (LF-type II) burst occurs significantly higher in the corona than the CME and its relationship to the CME is not straightforward. The analysis of the EIT wave (coronal bright front) shows the fastest wave component to be in the southeast quadrant of the Sun. This is also the quadrant in which the source positions of the LF-type II were found to be located, probably resulting from the interaction between the shock wave and a streamer.

Conclusions. The relationship between the CME/flare event and the shock wave signatures is discussed using the temporal association, as well as the spatial information of the radio emission. Further, we discuss the importance and possible effects of the frequently nonradial propagation of the shock wave.
\end{abstract}

Key words. Sun: radio radiation - magnetohydrodynamics (MHD) - shock waves - Sun: coronal mass ejections (CMEs) Sun: particle emission - Sun: heliosphere

\section{Introduction}

Large-scale energy release in the solar corona can appear in the form of coronal mass ejections (CMEs; e.g. Shibata \& Magara 2011; Green et al. 2018) and flares (e.g. Fletcher et al. 2011; Benz 2017). During these eruptive phenomena, particles are accelerated (Miteva et al. 2017), plasma is heated, and waves and shocks are generated (Aschwanden 2019). The shock waves associated with eruptive events can manifest via a variety of signatures. Chromospheric Moreton waves, EIT waves (coronal bright fronts associated with solar eruptions; see e.g. Zhukov \& Auchère 2004, and references therein), and type II radio bursts are often considered to be signatures of the same shock wave propagating in the solar corona (e.g. Warmuth et al. 2004; Vršnak et al. 2006; Veronig et al. 2006, 2010; Warmuth 2015 , and references therein). We focus on type II radio bursts which are the longest known signatures of shock waves in the solar corona (Wild 1950), and are also excellent means for track- ing the shock wave propagation (e.g. Wild \& McCready 1950; Ginzburg \& Zhelezniakov 1958; Melrose 1980; Klassen et al. 1999; Magdalenić et al. 2012).

Type III radio bursts (radio signatures of electron beams travelling along open and quasi-open magnetic field lines; see e.g. Reid \& Ratcliffe 2014) are also often observed in association with eruptive phenomena (e.g. Reiner et al. 1998, 2001; Cairns et al. 2003; Cremades 2007; Reid et al. 2014; Krupar et al. 2015). Both type II and type III bursts are generally considered to be plasma emissions generated by beams of suprathermal electrons. Type II and type III bursts are observed at both the fundamental plasma frequency $\left(f_{\mathrm{pe}}\right)$ and the second harmonic $\left(2 f_{\text {pe }}\right)$. Sometimes only one of the two components is observed (fora review, see e.g. Melrose 2017, and references therein). As the sources of radio emission propagate away from the Sun, radio emission occurs at progressively lower frequencies which corresponds to the decrease of the ambient electron density. Type II radio bursts observed at metric wavelengths (around $100 \mathrm{MHz}$ ) 
are generally considered to be signatures of shock waves propagating in the low solar corona while emission in the hectometric to kilometric wavelengths is associated with shock waves propagating through the outer corona to interplanetary space.

Understanding the origin of coronal shock waves and associated type II emission is a complex and widely discussed problem (e.g. Gary et al. 1984; Klein et al. 1999; Maia et al. 2000; Magdalenić et al. 2008, 2010; Nindos et al. 2011; Zimovets et al. 2012). Distinguishing the shock driver, particularly in the low corona, is often a difficult task, mostly due to the good temporal synchronisation between the flare impulsive phase and the acceleration phase of the CME. Although some shocks appear to be generated by flares (e.g. Magdalenić et al. 2010, 2014; Kumar et al. 2016; Eselevich et al. 2019), the majority of shock waves are CME driven (e.g. Vršnak \& Cliver 2008, and references therein). Even when the type II emission is clearly a signature of the CMEdriven shock wave, it is unclear whether the emission originates from close to the CME flank or the CME nose, that is, the relative position of the type II sources and the shock driver is unclear. A number of studies have demonstrated that the radio emission is most probably originating from the regions close to the CME flank (Reiner et al. 1998; Magdalenić et al. 2012, 2014; Shen et al. 2013; Martínez-Oliveros et al. 2015; Krupar et al. 2016, 2019). Only occasionally are events reported with the type II emission situated close to the CME nose regions (Martínez-Oliveros et al. 2012a; Mäkelä et al. 2016, 2018). Similarly, it was shown that coronal EIT waves are initiated by the fast acceleration of the CME flanks (Veronig et al. 2008, 2018; Kienreich et al. 2009; Patsourakos \& Vourlidas 2009; Long et al. 2017).

In this paper, we present a study of a CME/flare event between September 27 and 28, 2012 (referred to here as the September 27/28 event), and the associated radio event. We investigate the complex relationship between the CME, the shock wave, and the origin of the two associated type II radio bursts. We discuss the importance of the effects induced by the non-radial propagation of the CME-driven shock wave and the consequences for the associated radio emission. The observations employed in the study are introduced in Sect. 2, followed by the description of the CME/flare event (Sect. 3) and its propagation (Sect. 4). The study of the EIT wave associated with the eruption is presented in Sect. 5. The radio event is reported and analysed, employing the classical method, in Sect. 6, which is followed by the results of the radio triangulation study in Sect. 7 . An interpretation of the results with regards to the ambient coronal conditions is discussed in Sect. 8. The study is briefly summarised and the effects of radio wave propagation are discussed in Sect. 9, and finally, the most important findings of the study are listed in Sect. 10, respectively.

\section{Observational data}

The multi-wavelength study of the September 27/28 event employs white light (WL), radio, extreme ultra violet (EUV), magnetogram, and X-ray observations.

\subsection{White light coronagraph observations}

We used coronagraph observations from different instruments and viewpoints: (a) The Large Angle and Spectroscopic Coronagraph (LASCO; Brueckner et al. 1995) on board the Solar and Heliospheric Observatory (SOHO; Domingo et al. 1995) mission which provides two coronagraphs, C2 and C3, with different fields of view, and (b) and the Solar TErrestrial RElations Observatory Ahead and Behind (STEREO A and STEREO B; Kaiser et al. 2008) coronagraphs COR 1 and COR 2 (Howard et al. 2008).

\subsection{Extreme ultraviolet, magnetogram, and X-ray observations}

Observations of the Sun at the EUV wavelengths are often used in studies of the evolution of active regions, flares, waves, and on-disc signatures of CMEs. In this study we employed observations from two instruments: (a) The Extreme Ultra Violet Imagers (EUVI; Howard et al. 2008) instrument on-board STEREO which observe the solar corona with a cadence of $15 \mathrm{~min}$ in four EUV passbands; and (b) the Atmospheric Imaging Assembly (AIA; Lemen et al. 2012) onboard Solar Dynamics Observatory (SDO; Pesnell et al. 2012) which routinely provides high-cadence, high-resolution EUV images of the Sun from Earth's orbit. Additionally, we also employ soft X-ray observations by the Geostationary Operational Environmental Satellite (GOES 15; Garcia 1994).

\subsection{Radio observations}

In this study we used observations from the following groundand space-based observatories: (a) Dynamic spectra from Bruny Island Radio Spectrometer (BIRS; Erickson 1997), covering the decametric range $(80-20 \mathrm{MHz})$; (b) dynamic spectra from Culgoora, which cover the metric and decametric range (1800-18 MHz); (c) dynamic spectra from the STEREO/WAVES instruments (Kaiser 2005; Kaiser et al. 2008; Bougeret et al. 2008), which are routinely available in the frequency range $2.5-16025 \mathrm{kHz}$, and the high-frequency receiver (HFR), which provides instantaneous direction finding measurements at a number of discrete frequencies in the range 125-1975 kHz (Cecconi et al. 2008); and (d) dynamic spectra from the Wind/WAVES (Bougeret et al. 1995) instrument, which are available in the frequency range $4-13825 \mathrm{kHz}$, and the RAD1 receiver, which provides direction finding measurements (at selected frequencies; 100-1040 kHz).

\section{Event description}

The GOES C3.7 flare (23:35-23:47-1:40 UT) was associated with a two-step filament eruption and a full-halo CME first observed in the SOHO/LASCO C2 field of view at 00:00 UT on September 28, 2012 (Figs. 1a and c, respectively; studied in Veronig et al. 2019). We also observed on-disc signatures of the CME in the form of a coronal dimming and an EIT wave. The CME/flare event originated from NOAA active region 11577 (N09,W31), the photospheric magnetic field of which had a $\beta \gamma$ configuration at the time of eruption. The flare and the off-limb signatures of the CME were observed by SDO/AIA, together with a well-defined EIT wave propagating mainly in the southeast direction from the active region (best observed in the at AIA $193 \AA$ filter, Fig. 1b). The STEREO A/COR 2 and STEREO B/COR 2 coronagraphs observed the CME for the first time at 00:12 UT and 01:03 UT, respectively. The CME was also observed by both STEREO A and STEREO B Heliospheric imagers (HI) and is included in the Heliospheric Cataloging, Analysis and Techniques Services catalogue (HELCATS ${ }^{1}$ ). A WL shock wave observed by all three coronagraphs accompanied the CME under study.

1 https://www.helcats-fp7.eu/ 

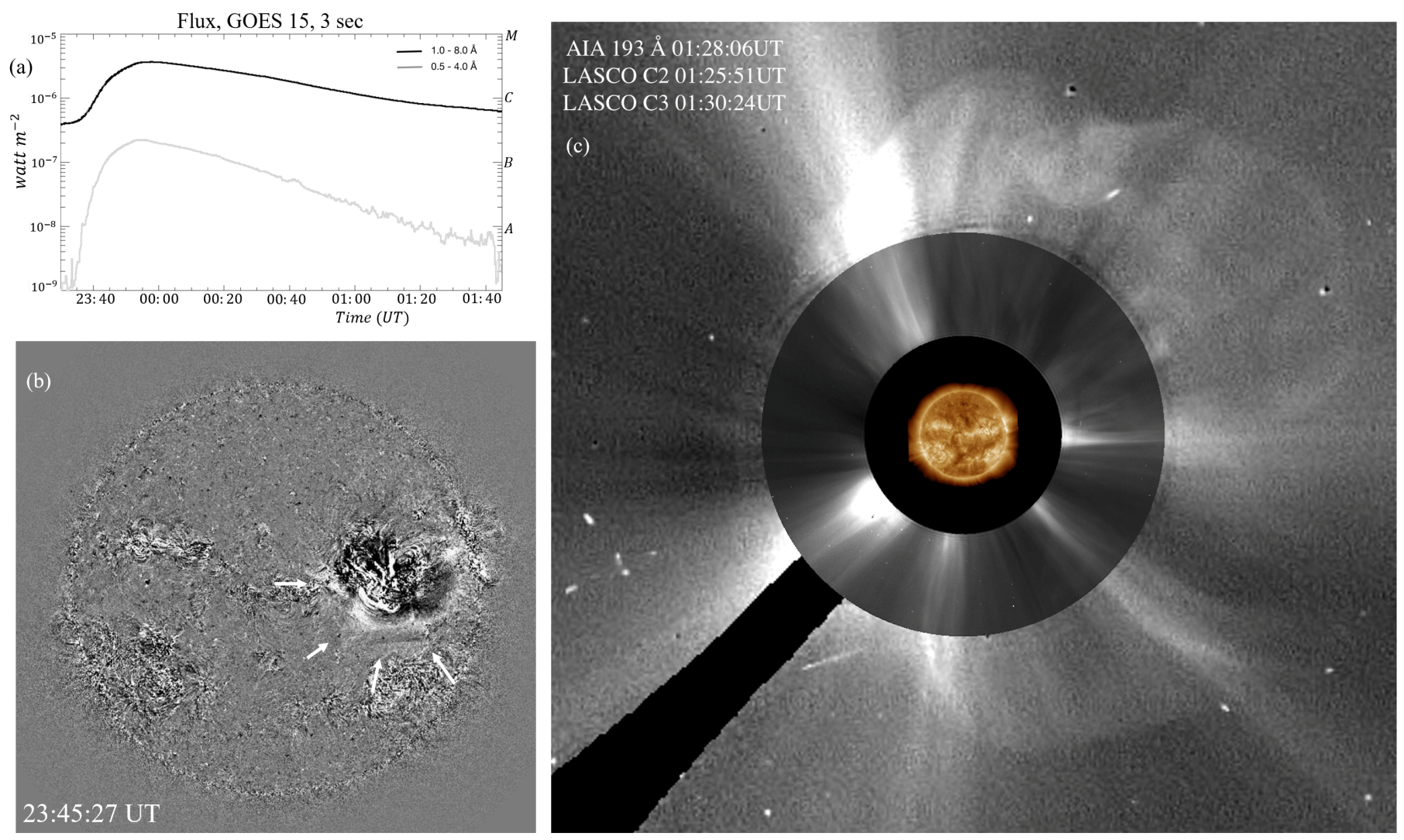

Fig. 1. Overview of the CME/flare event: Panel a: the GOES C3.7 X-ray flare curve shows a rather long flare decay phase. Panel $b$ : running difference image of the SDO/AIA $193 \AA$ channel. The white arrows mark what appears to be an EIT wave. Panel c: combined images of the SDO/AIA observations at $193 \AA$ and the SOHO/LASCO C2 and C3 observations around 01:30 UT.

In order to understand the possible preconditioning of the solar corona, that is, the possible existence of large preceding eruptions which could have perturbed the global structure of the corona and influenced the propagation of the CME and associated shock (e.g. Liu et al. 2014; Temmer \& Nitta 2015), we investigate the solar events in a time window of $12 \mathrm{~h}$ previous to the studied event. The only CME/flare event (hereafter, event-0) possibly associated with the one studied here was a back-sided halo-CME originating from NOAA AR 11574. Two subsequent eruptions were observed in the STEREO A/COR 1 field of view, starting shortly before 10:00 UT on September 27, and they were accompanied by an intense radio event. The two STEREO spacecraft observed the radio event across the entire frequency range, while Wind/WAVES observations show only the low-frequency part of the radio emission (the high-frequency part was occulted by the Sun). This back-side event could not have significantly influenced the development and the propagation of the main event studied here. The particularity of the radio event- 0 will be discussed in a separate publication.

\section{Propagation of the CME}

In order to study the CME kinematics, we use measurements of the CME height derived from STEREO A/EUVI, COR 1, and COR 2 images (Fig. 2a). STEREO A had the best view to observe the CME evolution close to the limb, thus minimising projection effects on the derived kinematic profiles. Figure 2 shows the CME kinematics along the position angle of $65^{\circ}$. The CME velocity (Fig. 2b) and acceleration (Fig. 2c) profiles were obtained by smoothing the height-time data and deriv- ing the first and second time derivatives (Dissauer et al. 2019). The smoothing algorithm that we use for approximating the curves (Podladchikova et al. 2017) was extended toward nonequidistant data. From the obtained acceleration profiles, we interpolate to equidistant data points based on minimisation of the second derivatives, and reconstruct the corresponding velocity and height profiles by integration. The projected speed of the CME leading edge reaches a peak value of $1490 \mathrm{~km} \mathrm{~s}^{-1}$, and the CME acceleration peaks on September 27 at 23:43 UT with a value of $860 \mathrm{~m} \mathrm{~s}^{-2}$.

\section{Modelling of the CME with EUHFORIA}

In order to understand the relationship between the CME and the shock wave, we modelled the CME using two complementary approaches: forward modelling using the graduated cylindrical model (GCS) model, and magnetohydrodynamics (MHD) modelling using EUropean Heliospheric FORecasting and Information Asset (EUHFORIA, Fig. 3; Pomoell \& Poedts 2018).

We apply the GCS model, a simple geometric reconstruction technique developed by Thernisien et al. (2006, 2009), using coronagraph images from multiple viewpoints, i.e. SOHO/LASCO C 2 and C3, and STEREO A and B COR 2. This technique is based on fitting the observed white-light structure of the CME using a croissant-like three-dimensional shell which, when applied to a sequence of imaging observations, allows us to determine the kinematic and geometric properties of the CME. These are then used as input for the heliospheric MHD simulations. Figures $3 \mathrm{a}-\mathrm{c}$ shows the results of the reconstruction of the CME on September 28 around 01:24 UT, resulting in the following CME parameters (in Stonyhurst coordinates): CME 


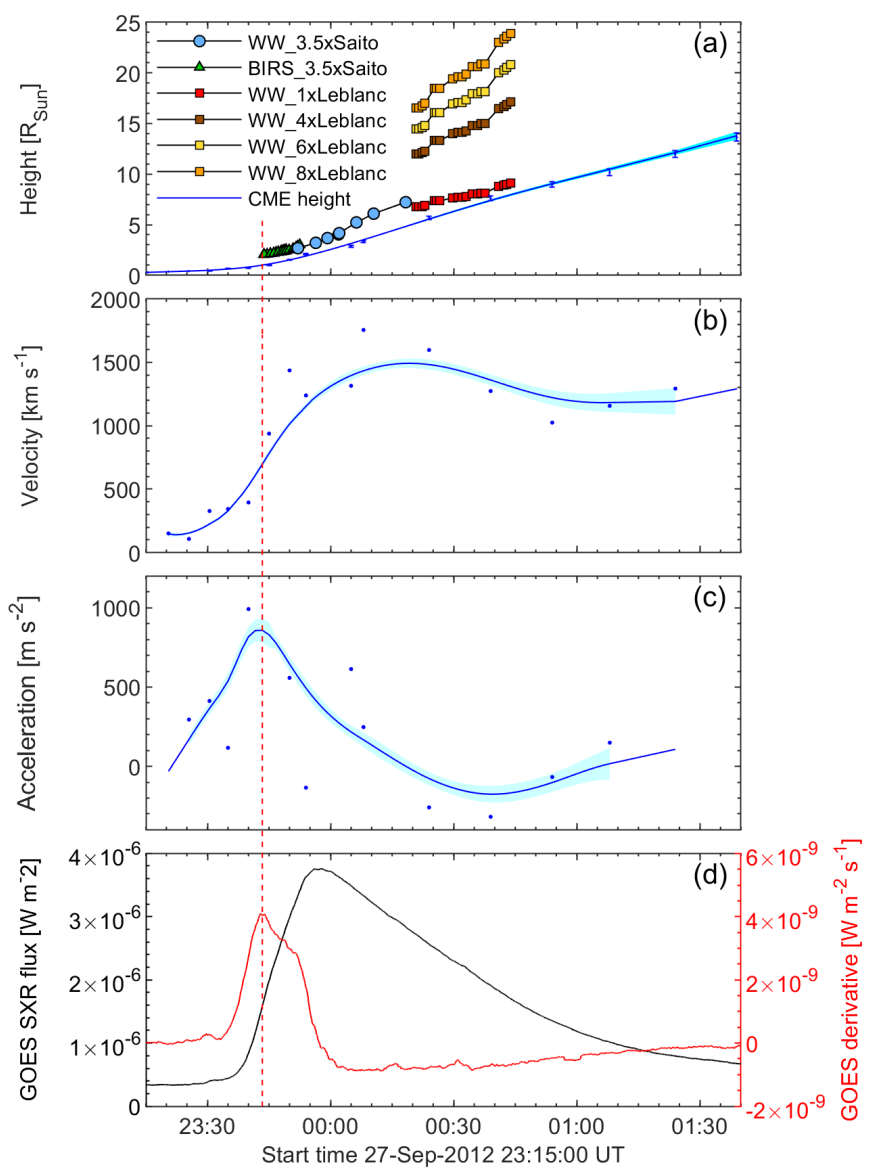

Fig. 2. CME kinematics based on STEREO A/EUV and coronagraph images. Panel a: CME height (blue dots) together with error bars and radio emission heights. The corresponding line shows the smoothed height-time profile. HF-type II heights are obtained using a 3.5-fold Saito, while the LF-type II heights are obtained using 1-fold, 4-fold, 6fold, and 8-fold Leblanc based on radio triangulation results discussed in Sect. 9. Panel b: CME velocity and panel $c$ : acceleration profile obtained by numerical differentiation of the data points (circles) and the smoothed curves (lines). The shaded areas represent the error ranges obtained from the smoothed curves. Panel $d$ : GOES soft X-ray flux and it's derivative. The red vertical line denotes the peak of the GOES derivative

latitude $\theta_{\mathrm{CME}}=20^{\circ}$, longitude $\phi_{\mathrm{CME}}=30^{\circ}$, front height $h_{\mathrm{CME}}=$ $11.9 R_{\odot}$, aspect ratio $\kappa_{\mathrm{CME}}=0.30$, leg angle $\alpha_{\mathrm{CME}}=20^{\circ}$, and tilt $\gamma_{\mathrm{CME}}=-90^{\circ}$. The estimated 3D speed of the CME is found to be $1270 \mathrm{~km} \mathrm{~s}^{-1}$ at 01:24 UT, which is comparable to the previous estimation of the CME speeds obtained in Sect. 2.1 from STEREO A/COR 2 images.

We use the EUHFORIA ideal-MHD heliospheric model to study the CME propagation. The simulations were performed using the EUHFORIA v1.0.4 version of the model (Hinterreiter et al. 2019) The CME parameters such as the half width, direction of propagation (longitude and latitude), and 3D speed obtained from the GCS reconstruction were used as input for the cone CME model (Odstrčil et al. 1996; Odstrčil \& Pizzo 1999; Pomoell \& Poedts 2018; Scolini et al. 2018). The predictive capabilities of EUHFORIA were already described in Pomoell \& Poedts (2018), Scolini et al. (2019, 2020).

Figure $3 \mathrm{e}$ shows the modelled CME after its insertion in the heliospheric domain. The ecliptic and the meridional cuts of the modelled CME and the background solar wind are shown on the left and right hand sides of the figure, respectively. The CME then propagates self-consistently as a MHD disturbance (Fig. 3d). The speeds of the modelled cone CME plotted in Fig. $3 \mathrm{e}$ show that the fastest component is close to the CMEflank regions.

\section{The EIT wave}

We studied the kinematics of the EIT wave associated with the CME using high-cadence EUV imagery obtained by the SDO/AIA $193 \AA$ filter. We derived the location and strength of the wave crest by calculating the intensity perturbation profiles from running difference image sequence using the ring analysis method (Podladchikova \& Berghmans 2005; Podladchikova et al. 2019). We first constructed a spherical polar coordinate system with its centre on the brightest part of the associated flare, called the "eruptive centre" (see e.g. Warmuth et al. 2004). The image was then divided into rings of equal width around the eruptive centre. We defined five angular sectors, where the EIT wave propagation is most pronounced (Fig. 4a). Sectors 1 and 2 cover the regions of direct wave propagation, that is, where it propagated without interactions, while sectors 3-5 are disturbed by strong interactions with ARs (Active regions) and the southern polar coronal hole. For each sector, we derived intensity perturbation profiles by calculating the mean intensity with the chosen binning of the rings. The outer border of every ring element is related with the corresponding distance from the source region. As a result, we obtained the projections of the radial intensity profiles onto the surface along the line of sight of SDO.

Figures $4 \mathrm{~b}$ and $\mathrm{c}$ shows the dependence of the EIT wave amplitude on the distance from the eruptive centre in sectors 2 and 4, respectively. Close to the source region, we observe areas of minimal intensity, that is, coronal dimming (studied in Veronig et al. 2019), which results from the density depletion caused by the evacuation of plasma during the CME lift-off (e.g. Hudson et al. 1996; Thompson et al. 1998; Dissauer et al. 2018). The EIT wave front is characterised by a sharp increase of the intensity towards its maximum (wave crest) followed by a decay to the background level. We identified the location of the wave crest (indicated by dots in Figs. $4 b$ and c) over the period of EIT wave propagation from the eruptive centre towards the solar limb. The obtained mean velocity of the EIT wave in sectors 1 and 2, which are undisturbed by ARs, is 500 and $360 \mathrm{~km} \mathrm{~s}^{-1}$, respectively. In sector 3 , we observe a further decrease of the velocity to $310 \mathrm{~km} \mathrm{~s}^{-1}$. However, when passing through AR 11576 situated south of the source region, the EIT wave velocity doubled its value to about $720 \mathrm{~km} \mathrm{~s}^{-1}$. This increase in the EIT wave speed is most probably related to the higher local Alfvén speed in regions of strong magnetic fields of the AR (e.g. Mann et al. 1999a).

A similar profile is observed in sector 4 (Fig. $4 \mathrm{c}$ ), where the EIT wave propagated with a mean velocity of $320 \mathrm{~km} \mathrm{~s}^{-1}$ and accelerated to a speed of $770 \mathrm{~km} \mathrm{~s}^{-1}$ while passing through the southern AR. In sector 5, the wave is observed only as a relatively diffuse structure. The average speed of the EIT wave was found to be about $280 \mathrm{~km} \mathrm{~s}^{-1}$. The results from the study of the EIT wave were used to reconstruct the coronal shock wave and study its propagation in Sect. 8.2.

\section{Radio event}

The radio event associated with the studied CME/flare event was observed by both ground- and space-based instruments. The 

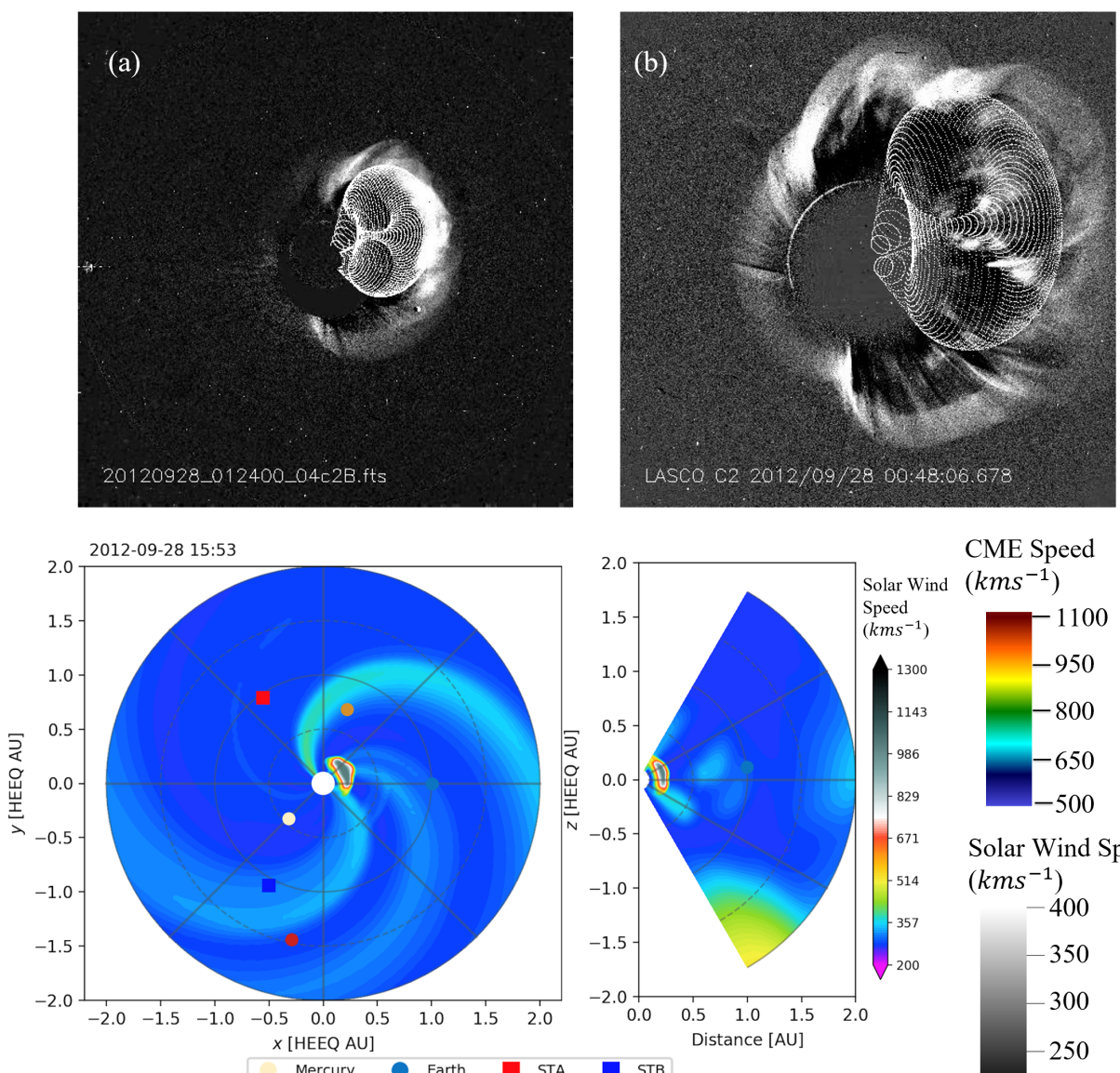

(d)

.

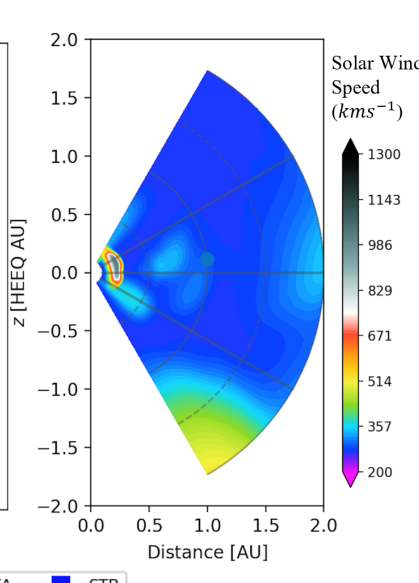

CME Speed

$\left(k m s^{-1}\right)$

$(k m s$
-400

$-350$

$-300$

$-250$

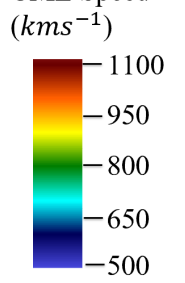

Solar Wind Speed
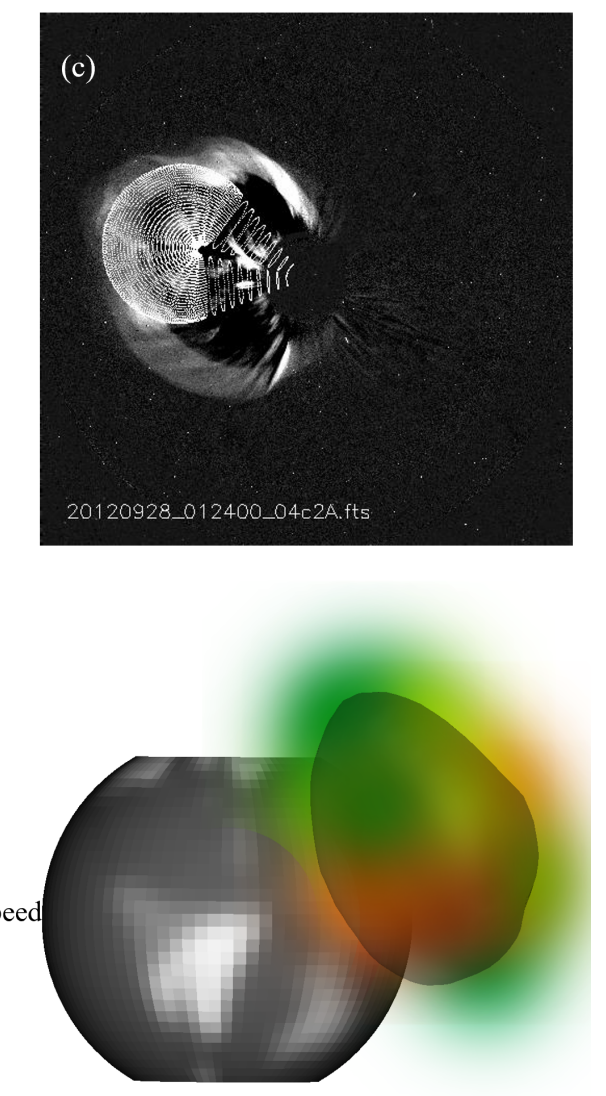

(e)

Fig. 3. Panels $a-c$ : graduated cylindrical shell (GCS) reconstruction of the CME body. Coronagraph images from: $(a)$ STEREO B/COR 2, (b) SOHO/LASCO C3, and (c) STEREO A/COR 2 at roughly the same time (September 28, 2012 at 01:24 UT). Panels $d$ and $e$ : CME modelled by EUHFORIA. $(d)$ Propagation of the modelled cone CME in the ecliptic and meridional perspectives. (e) CME speeds at the cone surface as seen from Earth. The grey sphere shows the solar wind radial velocity at the inner boundary of EUHFORIA (0.1 AU).

BIRS dynamic spectra (Fig. 5) show a structured and patchy metric type II burst which continues into the hectometric range as observed by the Wind/WAVES and STEREO/WAVES instruments (Fig. 6). This high-frequency type II burst (hereafter, HFtype II) was observed from about 23:43 UT on September 27 until about 00:30 on September 28. A second type II burst was observed only by space-based instruments due to its low starting frequency of $2000 \mathrm{kHz}$ for Wind/WAVES, and $1000 \mathrm{kHz}$ as observed by the STEREO/WAVES instruments. This lowfrequency type II (hereafter, LF-type II) was observed in the time interval 00:05-00:50 UT on September 28. Both the HF and LFtype II bursts show fundamental and second harmonic emission lanes. The LF-type II was observed by all three WAVES instruments (on board Wind, STEREO A, and STEREO B). Groups of type III bursts (see Fig. 6) were also observed during the flare impulsive and decay phase (hereafter, FI-type III and FDtype III) by all three WAVES instruments (during time interval 23:20-01:30), but with different starting frequencies as seen from different spacecraft. We note that the high-frequency observations of STEREO B/WAVES (up to $2 \mathrm{MHz}$ ) do not show any radio emission. Taking into account the spacecraft position at the time of the event (STEREO A and STEREO B separated by $125^{\circ}$ and $-118^{\circ}$ from Wind) and knowing that the source region of the $\mathrm{CME} /$ flare event was on the back side of the solar disc as observed by STEREO B, we conclude that the radio emission was occulted for it.
In order to obtain the type II kinematics, we employed the classical method (e.g. Magdalenić et al. 2008, 2010, 2014) using the drift rate of the radio bursts and coronal electron density models. The Saito (1970) and Leblanc et al. (1998) electron density models are two of the most frequently employed 1D density models for the metric and decametre to hectometre frequency range, respectively. Similar to some previous studies we employ a 3.5-fold Saito density model for metric observations (e.g. Magdalenić et al. 2008, 2010) and a 1-fold Leblanc density model for the decametre to hectometre range (e.g. Palmerio et al. 2019). Figure 7 shows the type II drift rates estimated by considering the central part of the emission band. Type II speeds obtained with this method for the HF-type II and the LF-type II are about $1500 \mathrm{~km} \mathrm{~s}^{-1}$ and $1000 \mathrm{~km} \mathrm{~s}^{-1}$, respectively. The error bars in Fig. 7 show the uncertainty of the obtained results. Figure 7 indicates that the LF-type II burst is the continuation of the HF-type II burst. However, their strongly different positions in the dynamic spectra (Fig. 6) do not support this conclusion. In Fig. 2a, we present the LF-type II kinematics employing three different $1 \mathrm{D}$ density models in order to highlight the drastic change in interpretation of the radio emission induced by the different models.

A more accurate method to estimate the shock wave kinematics is the so-called radio triangulation method, which employs direction finding observations (Sect. 7). However, as the direction finding observations are not always available, we 

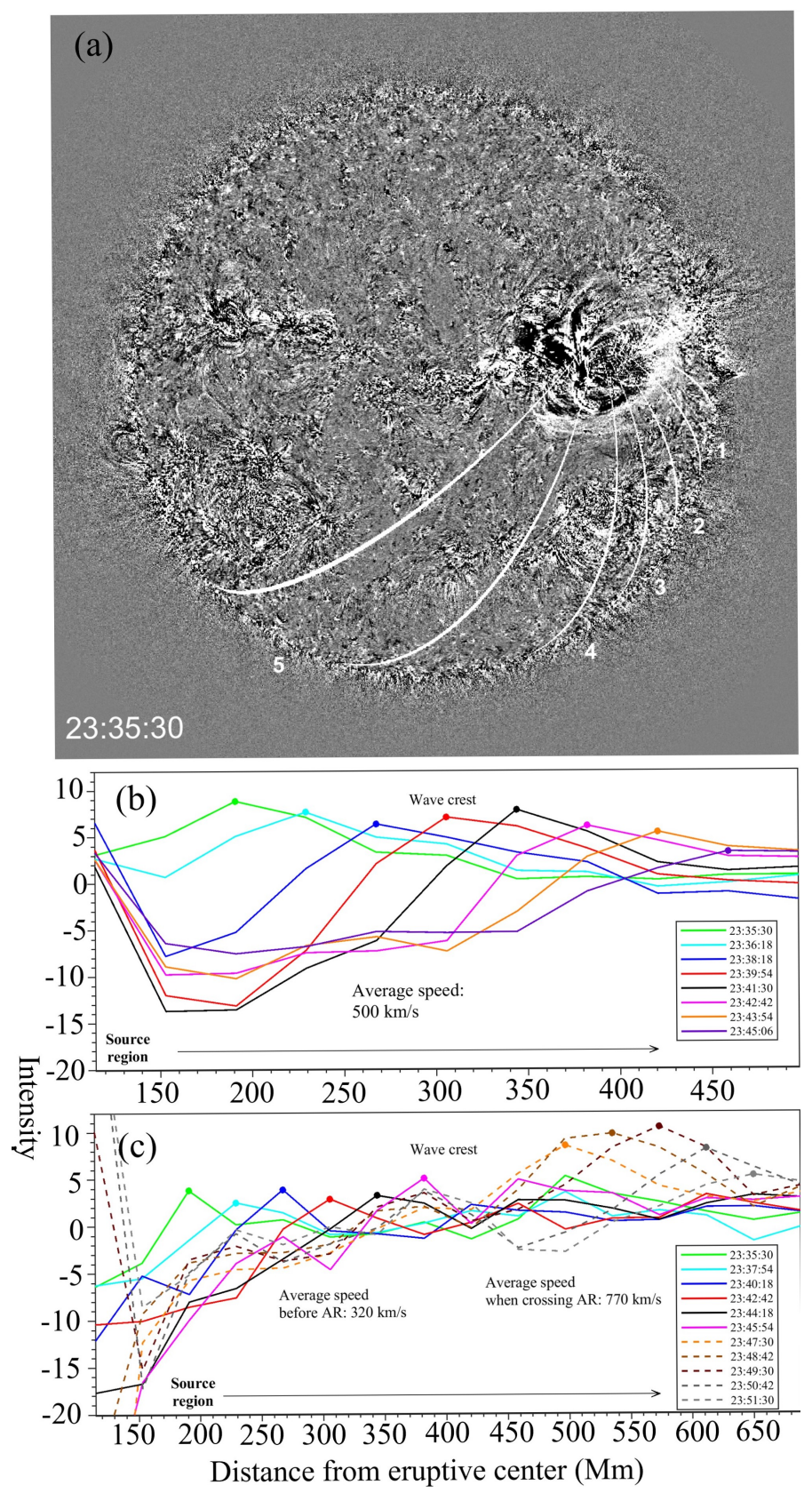

Fig. 4. Panel $a$ : EIT wave observed by the SDO/AIA $193 \AA$ filter. The region where the EIT wave was most pronounced is divided into five sectors. Panel b: evolution of the EIT wave profiles in sector 2, revealing a fast decay. Panel $c$ : evolution of the EIT wave in sector 4 .

first discuss the radio event qualitatively. A more quantitative method using radio triangulation is presented in Sect. 7.2. The Wind/WAVES observations (Fig. 6, bottom panel) show both fundamental and the second harmonic lanes of intense LF-type II burst. A qualitative assessment indicates that the intensity of the radio burst is strongest in Wind/WAVES, is somewhat fainter in the STEREO B/WAVES, and is very faint in the STEREO A/WAVES observations (middle and top panel of Fig. 6, respectively). Taking into account the assumption that the radio emission is most intense in the direction of its propagation (like e.g. in Magdalenić et al. 2014), we can roughly deduce the direction of the shock wave propagation to be between STEREO B and Wind, and somewhat closer to the Wind spacecraft. Although this is only a qualitative assessment, it can provide additional

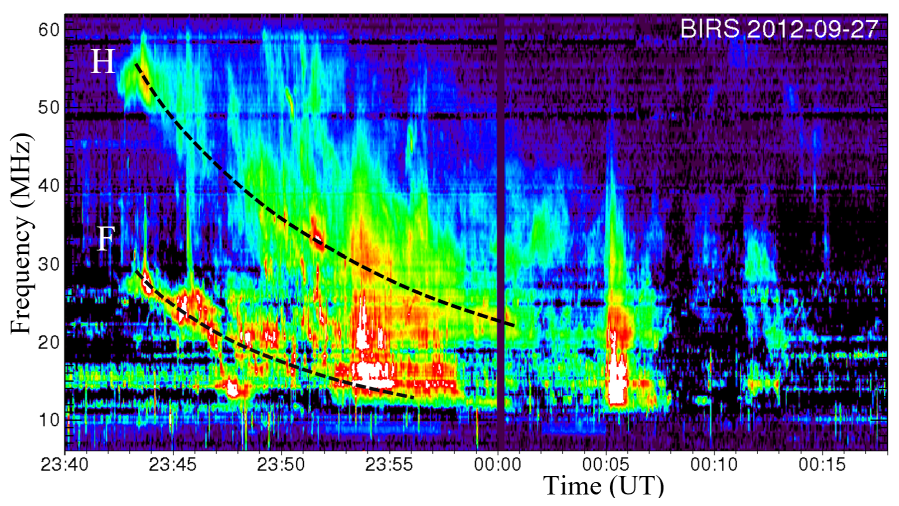

Fig. 5. Dynamic spectra (observed by BIRS) show radio emission in decametric and hectometric range. The dotted lines indicate the trend of the patchy type II burst observed at both fundamental and harmonic emission.

information in a case where only the classical method for estimation of the shock wave kinematics is possible.

\section{Radio triangulation}

The kinematics obtained from type II drift rate using radial density models, such as the one presented in Fig. 2a, are useful but do not provide information on the spatial position of the radio sources. Therefore, we use the unique method for estimating the 3D positions of the radio sources in the interplanetary space, namely the radio triangulation technique. This technique has so far mostly been used to study type III radio bursts (Fainberg et al. 1972; Gurnett et al. 1978; Reiner \& Stone 1988), and has only recently been more frequently used to study type II bursts (Hoang et al. 1998; Reiner et al. 1998; Martínez-Oliveros et al. 2012a, 2015; Magdalenić et al. 2014; Krupar et al. 2016, 2019; Mäkelä et al. 2016, 2018). Depending on the type of spacecraft, i.e. spinning or three axis stabilised, we distinguish different direction finding techniques (e.g. Fainberg \& Stone 1974; Lecacheux 1978; Manning \& Fainberg 1980; Santolík et al. 2012; Cecconi \& Zarka 2005; Krupar et al. 2012; Martínez-Oliveros et al. 2012b). For Wind (spinning spacecraft) observations we employed a spin demodulation technique (Fainberg \& Stone 1974) and for STEREO (three axis stabilised spacecraft) observations we employed a singular value decomposition technique (Krupar et al. 2012). The radio triangulation studies are performed using simultaneous direction finding observations of at least two spacecraft.

The radio triangulation analysis in this study was done employing the following premises:

- The direction finding observations are available for a selected set of frequency channels at each spacecraft. The observing frequencies of STEREO and Wind are slightly different (Bougeret et al. 1995, 2008) which might induce uncertainty in the radio triangulation results. Similar to previous studies (e.g. Martínez-Oliveros et al. 2012a, 2015; Magdalenić et al. 2014; Mäkelä et al. 2016, 2018), in order to combine Wind and STEREO observations, we considered the closest frequency pairs.

- The direction finding technique provides wave vectors which are used in the radio triangulation studies to estimate the 3D radio source positions. As previously done by Magdalenić et al. (2014), we use the full distance between the two wave vectors at a given frequency pair as the radio source 


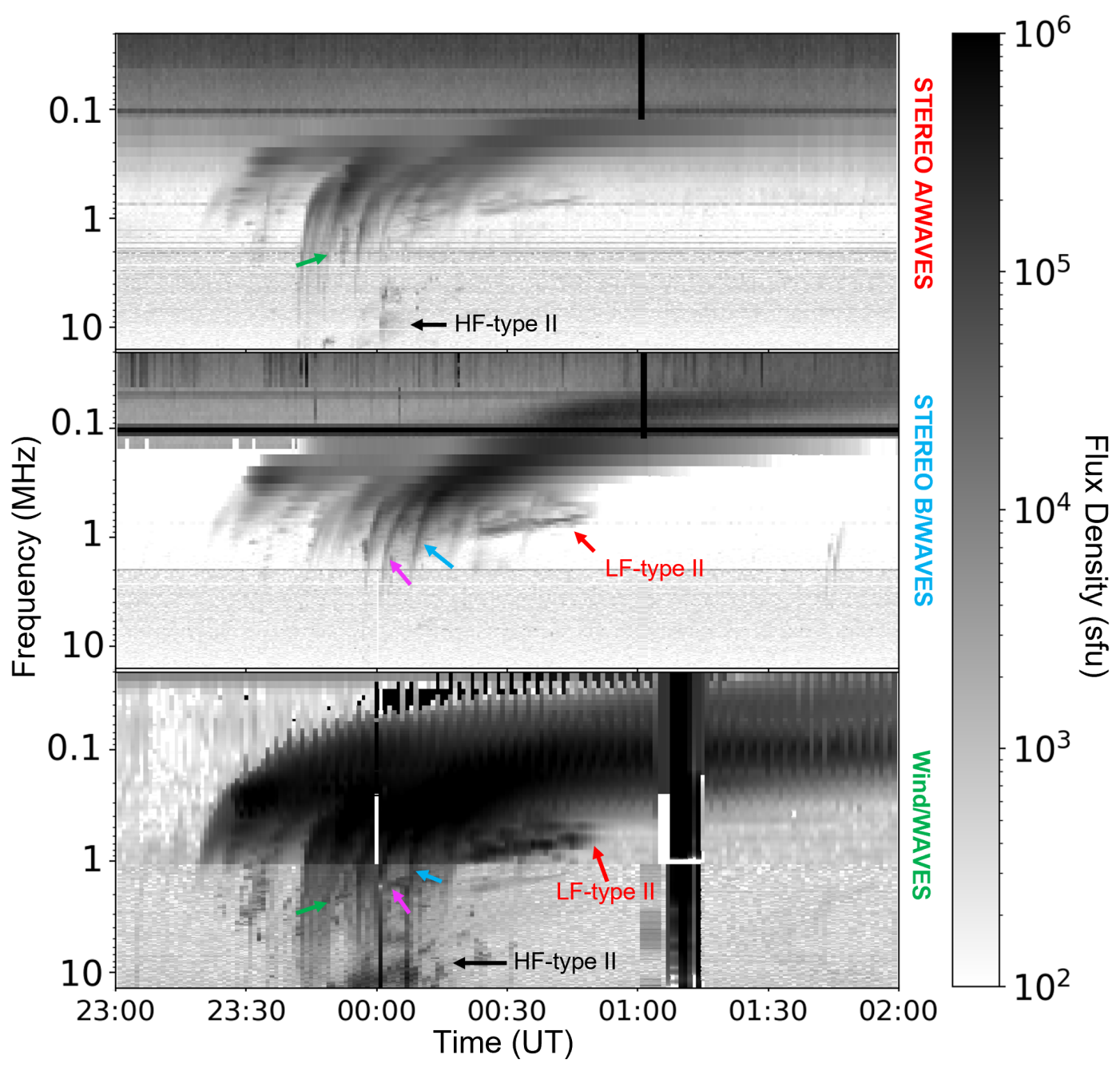

Fig. 6. Calibrated dynamic radio spectra (solar flux units, i.e. $s f u$ ) observed by the Wind/WAVES, STEREO A/WAVES, and STEREO B/WAVES show the radio event associated with the September 27/28 event flare/CME. The LF-type II (indicated by the red arrow) burst observed by all three spacecraft, shows fundamental and second harmonic emission lanes. The HF-type II burst which is indicated by the black arrow was best observed by Wind/WAVES. The flare impulsive (FI) type III group (marked by the green arrow) and the type III associated with the flare decay (FD and $\left.\mathrm{FD}^{*}\right)$ phase are indicated by the blue and pink arrows, respectively.

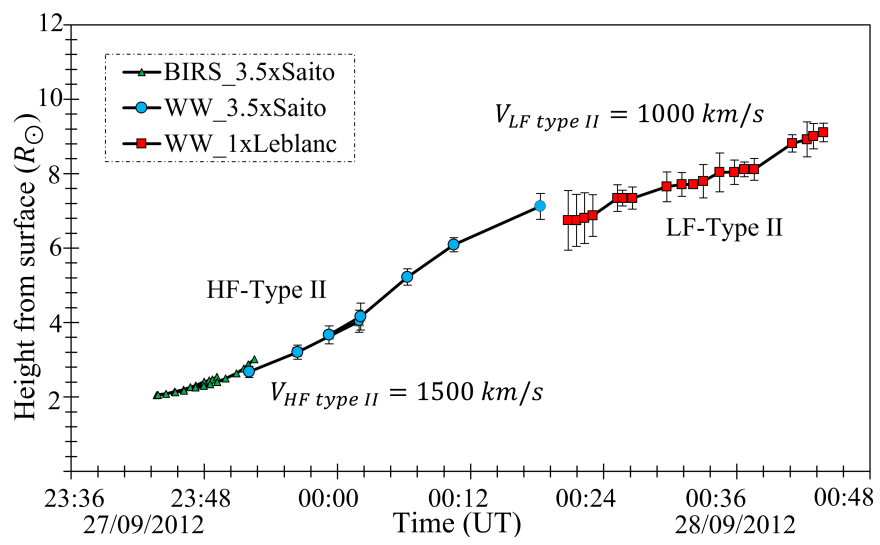

Fig. 7. Kinematics of the shock wave obtained using the type II burst frequency drift and coronal electron density models. The HF-type II was observed by both ground- and space-based instruments, while the LF-type II was observed only by space-based instruments. The HFtype II heights are obtained by using a 3.5 -fold Saito density model, and the LF-type II heights are obtained using a 1-fold Leblanc density model. The spectral range of the bursts is emphasised by the error bars. region. This region is then presented in the figures as a sphere, with a diameter equal to the distance between the wave vectors. We note that the intrinsic geometric errors of the radio triangulation technique are quite large regardless of frequency, and they are mostly due to the receiver gain and the position of the spacecraft pair (see Krupar et al. 2012, for more details). Therefore, we do not discuss the geometric radio source sizes, but only the radio source regions as defined above. A similar procedure was used in previous studies (Reiner et al. 1998; Martínez-Oliveros et al. 2012a, 2015; Mäkelä et al. 2016, 2018; Krupar et al. 2020).

- The estimated distance between the two wave vectors is generally smaller at high frequencies. Therefore, in this analysis we used the highest available frequencies of direction finding observations, and did not use frequencies below $500 \mathrm{kHz}$.

- The points for radio triangulation studies were selected taking into account the time delay which is due to different travel times needed for a radio signal to arrive at the different spacecraft. The magnitude of the time delay is not absolute as it depends on the direction of propagation of the radio emission. 


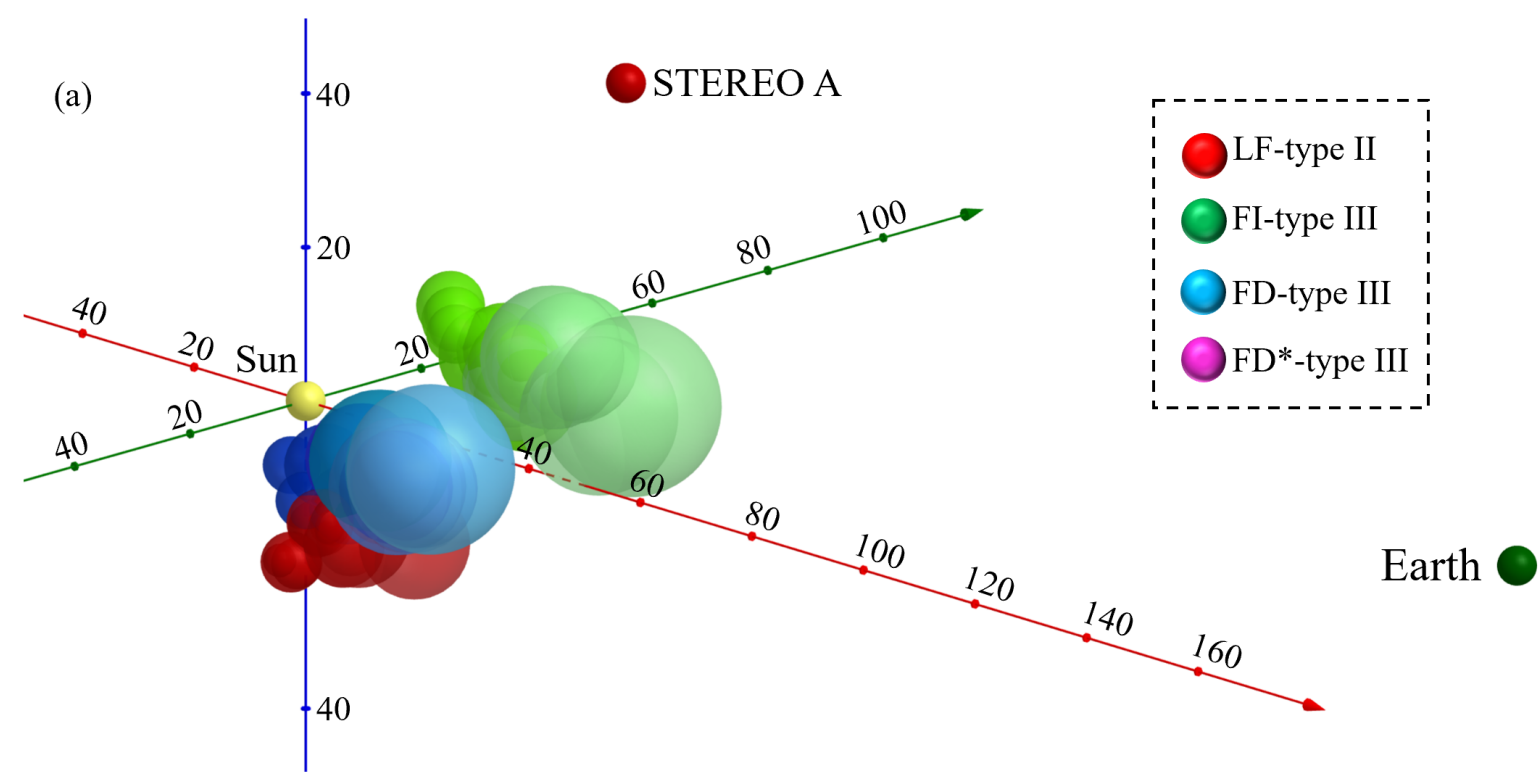

(b)

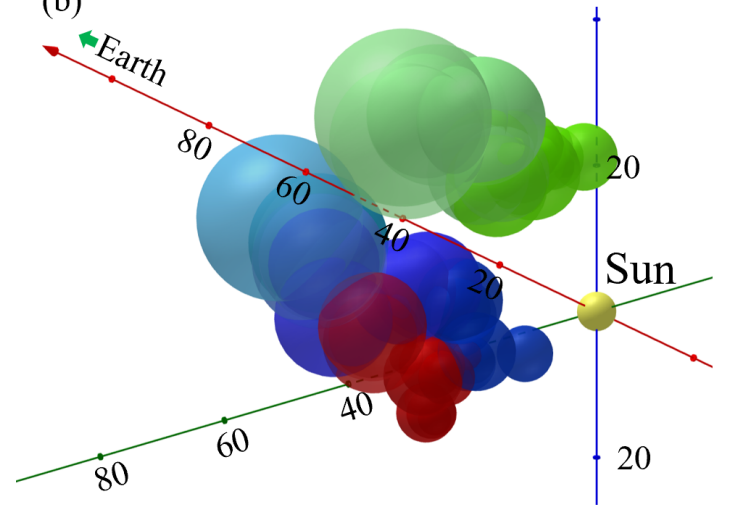

(c)

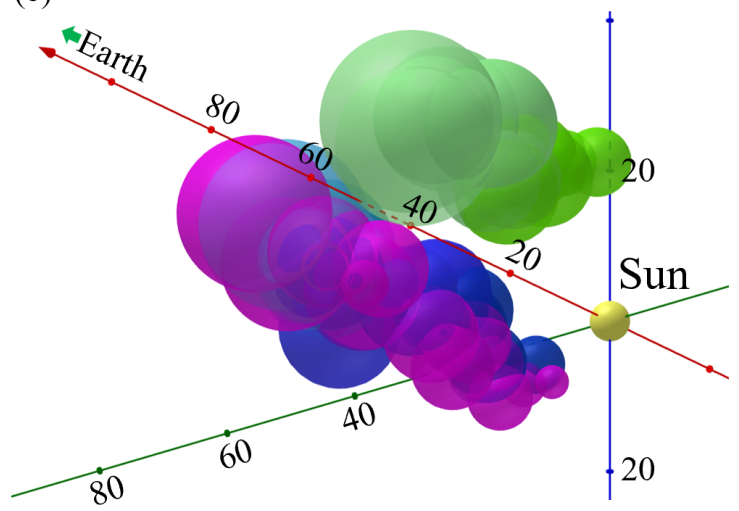

Fig. 8. Panel a: results from radio triangulation studies of type II and type III radio bursts. The yellow sphere represents the Sun, while the red and green spheres represent STEREO A, STEREO B and Wind, respectively. The spheres of varying size represent the radio source regions. The green spheres mark the radio source positions of the FI-type III close to the CME flank. The LF-type II and the FD-type III, denoted by the red and blue spheres, have source positions at the southeast side of the Sun, i.e. close to another CME flank. Panel b: FI-type III, FD-type III, and LF-type II plotted from a different perspective. Panel $c$ : three different type IIIs plotted together.

- The intensity of the type II bursts is significantly lower than for the type III bursts, and so in order to have the radio emission sufficiently above the background level, similar to Magdalenić et al. (2014), we employed background subtraction of only $5 \%$ for all direction finding data.

Three combinations of direction finding observations are possible. The results obtained using STEREO A/WAVES and STEREO B/WAVES observations are unreliable due to large angular separation of the spacecraft. We present the results for the other two spacecraft pairs: STEREO A/WAVES and Wind/WAVES, and the STEREO B/WAVES and Wind/WAVES. These direction finding observations not only show the highest intensity of radio flux but also give the smallest distances between the wave vectors and the most reliable results.

\subsection{Source positions of type III radio bursts}

We distinguish two groups of type III bursts associated with the studied event. The first group are type III bursts temporally associated with the flare impulsive phase (FI), observed at about 23:52 UT (marked in Fig. 6 by green arrow). The second group are the two type III bursts associated with the flare decay phase (FD), observed at 00:00 and 00:10 UT (marked in Fig. 6 by blue and pink arrows). Similar to the qualitative analysis of the type II bursts, (see Sect. 6), we also discuss propagation of the type III bursts. If we consider that the radio emission is the most intense in the direction of its source propagation, and use only the dynamic spectra, we can deduce that the source region of FItype III appears closer to STEREO A than the source region of the FD-type IIIs (FD-type III and FD*-type III). Likewise, the source region of the FI-type III appears to be further away from STEREO B than the source region of the FD-type III bursts. In order to quantify the possibly different source positions of the FI-, FD-type III, and FD*-type III bursts, we performed radio triangulation.

In order to triangulate type III bursts, we used observations from two spacecraft pairs: (a) Wind/WAVES and STEREO A/WAVES; and (b) Wind/WAVES and STEREO B/WAVES. For all type III bursts, we considered the same frequency pairs (in $\mathrm{kHz}$ ): 525/548, 575/548, 625/624, 675/624, 725/708, 775/708, $825 / 804,925 / 916,1025 / 1040$ and 1075/1040, respectively.

The results of the triangulation are shown in Fig. 8a. The yellow sphere represents the Sun, and red, blue, and green 
spheres represent the three spacecraft STEREO A, STEREO B, and Wind, respectively. The source positions of radio bursts are colour coded. Darker colours denote sources situated closer to the Sun (high frequencies) and the lighter colours those further away from the Sun (low frequencies). The type II source positions are shown by red spheres and the type III source positions by green, blue, and pink spheres.

Figure $8 \mathrm{~b}$ shows that the sources positions as well as propagation path of the FD-type III and FD*-type III are significantly different from the FI-type III. The open field lines, along which the FD-type III and FD*-type III bursts propagate, are in the southwest quadrant of the Sun. The FI-type III bursts were observed in the northwest quadrant of the Sun. The change in the type III source positions happens at about the flare peak time. We note that the direction finding observations allowed us, for the first time, to quantitatively estimate the significantly different source positions of type III bursts associated with one CME/flare event.

\subsection{The low-frequency type II radio burst}

The radio triangulation study was only performed for the LFtype II burst because the HF-type II was not observed in the range of the direction finding frequencies (Fig. 6). For the analysis we selected the following frequency pairs (in $\mathrm{kHz}$ ): 575/548, 625/624, 675/624, 725/708, 775/708, 825/804, 875/804 and $925 / 916$ from STEREO B and Wind, respectively.

Figure 8a shows that the source positions of the LF-type II are situated in the southwest part of the Sun. The darker coloured circles represent high-frequency pairs positioned closer to the Sun. The close-up in Fig. 8 shows the slow drift of the type II emission from the south towards the solar central meridian. We also note that the LF-type II positions are roughly co-spatial with the positions of the FD-type III burst.

\subsection{Coronal electron density profiles and propagation direction of the radio emission}

The 3D source positions of the radio emission obtained from the triangulation study can be converted to radial distances (Fig. 9) and compared with the generally employed 1D coronal density models. The radio source positions are plotted in Fig. 9 together with 1D coronal electron density profiles (Saito 1970; Leblanc et al. 1998). The frequency $(f \propto n)$ is presented as a function of the radial heights. The horizontal bars denote distances between the two wave vectors.

The obtained density profiles along the propagation path of the LF-type II (Fig. 9a) and FD-type III (Fig. 9c) are similar, crossing different density models (from 3.5-fold Saito to 8-fold Leblanc). The similarity of the profiles is expected as the source regions of these bursts are propagating through the same region in the corona. The trend of crossing different density models is probably a consequence of the non-radial propagation of the radio source (Fig. 14). The FI-type III (Fig. 9b) shows a somewhat different profile, which is expected as the electron beam propagates along a magnetic field line on a different flank of the CME (Fig. 8). We note that all density profiles obtained from the radio triangulation study indicate unusually high values. We note that a tenfold Saito density model is used rarely and only in cases of large eruptions in the low corona $\left(\approx 1.5 R_{\odot}\right)$, such as for example that presented by Pohjolainen (2008). One of the processes which possibly influences the results of the radio triangulation is the scattering of the radio emission (see e.g. Melrose 1970; Thejappa et al. 2007; Kontar et al. 2019). We do not exclude that the absolute values of the densities obtained herein might be impacted by scat-
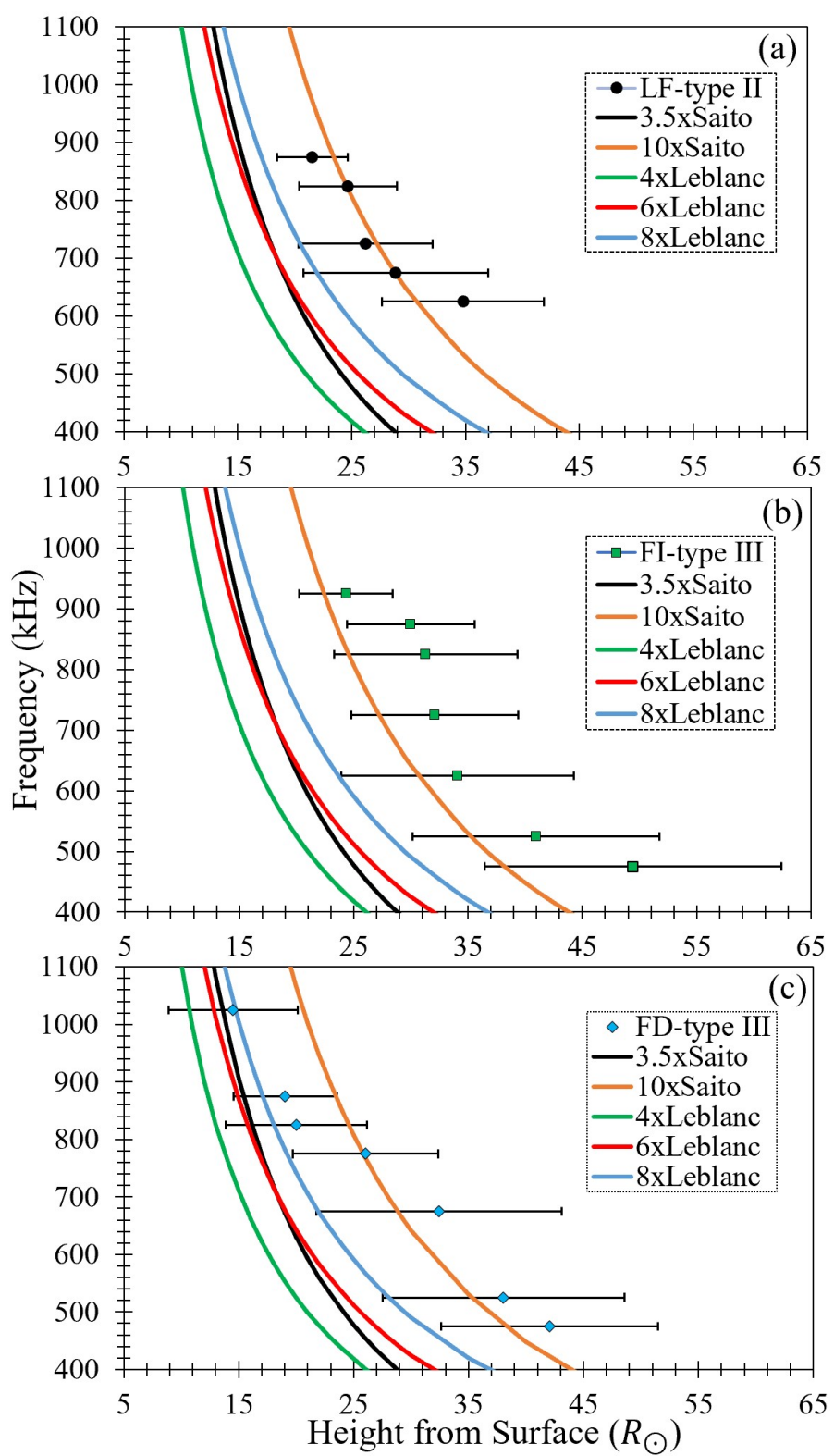

Fig. 9. Frequency shown as a function of heliocentric distance. Radio triangulation results are plotted together with two density models (Saito, Leblanc) for comparison. The markers show the source positions of three different radio bursts and the bars attached to them show the distance between the wave vectors. Panel $a$ : the black spheres show LFtype II, panel $b$ : FI-type III is noted by the green squares, and panel $c$ : the blue diamonds indicate the FD-type III.

tering effects. Although the scattering can indeed influence the observed radio source positions, it should not significantly affect the general direction of the propagation of the radio sources. We believe that the obtained results are mainly due to the non-radial propagation directions of the radio sources.

\section{Ambient coronal conditions and their influence on the eruptive event}

\subsection{Shock wave propagation through the corona: interaction with streamer}

We also investigated the possibility of the shock wave interactions with the ambient coronal structures. Figure 10 shows how EUHFORIA models the heliospheric current sheet in the time 


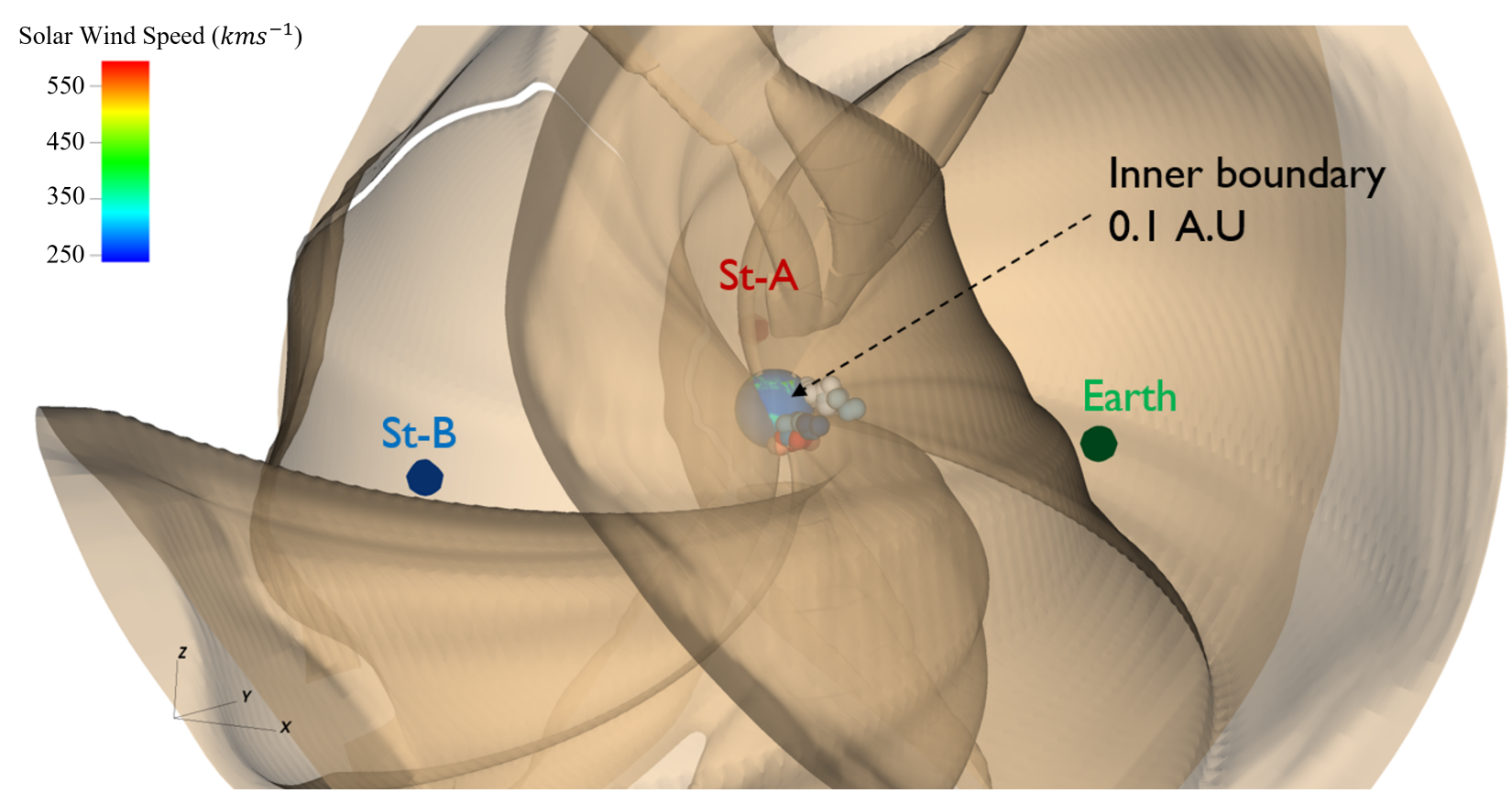

Fig. 10. Radio triangulation results plotted together with the isosurface of the heliospheric current sheet $\left(B_{r}=0\right)$ modelled by EUHFORIA. The radio sources of the LF-type II emission seem to cross the complex structure of the heliospheric current sheet several times.

of the studied event. The complex structure of the heliospheric current sheet (HCS) is not unusual during high levels of solar activity. Employing the coronagraph observations, we identified three streamers in the southeast quadrant of the Sun (Fig. 11a) that were perturbed by the passage of the shock wave. The bending of the streamers due to the shock wave propagation was particularly visible in white-light coronagraph observations by STEREO B/COR 1.

We find that the direction along which the LF-type II emission was located coincides with the direction of the fastest EIT wave component (Sect. 5, Fig. 4), that is, southeast from the source region. In order to understand the relative position of the LF-type II burst and the nearby coronal structures, in particular streamers (a preferable place for the generation of radio emission; Shen et al. 2013; Floyd et al. 2014), we projected the centre of the radio source region on the SOHO/LASCO C2 image. A white-light image recorded at 00:24 UT, shortly after the CME eruption, was selected. Most of the projected LF-type II sources were outside the range of the SOHO/LASCO C2 field of view (Fig. 11a). Therefore, for better comparison we mark the edges of the streamer stalk region at the heights beyond the SOHO/LASCO C2 field of view. Figure 11a shows that the projected positions of the centres of the radio source regions and the streamer stalk are close to each other.

We also performed a $3 \mathrm{D}$ reconstruction of the streamers in the southeast quadrant of the Sun using the tie-pointing method (e.g. Inhester 2006). Figure $11 \mathrm{~b}$ shows that the 3D positions of the reconstructed streamer are in agreement with the projected LF-type II source regions. We note that this way of projecting the type II sources, from 3D space to the 2D plane of sky, causes the sources to appear stationary. This effect is less visible in the Fig. 11a in which only the position of the source region centres is presented but not the full distance between the wave vectors. The above results, together with knowledge of the $3 \mathrm{D}$ positions of radio sources, allows us to suggest that the type II radio emission was enhanced by the interaction between the shock wave and the streamer, similar to previous studies (Feng et al.
2012; Magdalenić et al. 2014; Zucca et al. 2018; Mancuso et al. 2019).

\section{2. shock wave propagation through the corona: association with the EIT wave}

The EIT wave study (Sect. 5) shows that the speed of the EIT wave is greater when considering the directions from the source region towards the southern polar coronal hole (direction 2 and 3 in Fig. 4). If we assume that the EIT wave is the low coronal counterpart of the coronal shock wave (e.g. Mann et al. 1999b; Warmuth et al. 2005; Veronig et al. 2006; Muhr et al. 2010; Warmuth 2015), then the propagation direction of the fastest component of the EIT wave should roughly correspond to that of the fastest component of the CME and associated shock wave (as shown in Sect. 4, Fig. 3e). This region also coincides with the one where the LF-type II sources are situated.

In order to further inspect the association between the EIT wave and the type II bursts we performed a simple 3D reconstruction of the EIT wave (similar to Zucca et al. 2014, 2018; Rouillard et al. 2016) using parameters obtained in Sect. 5, and the global magnetic field configuration using a Potential Field Source Surface model (PFSS; Schrijver \& De Rosa 2003). In the presented model of the EIT wave in the 3D domain, we restricted our study to the heights of $2.5 R_{\odot}$ in order to avoid oversimplification of the wave dynamics at the larger heights as the anisotropic wave expansion might result in a wave deformation (Temmer et al. 2011). Due to these height restrictions, the modelled results are constrained to the low corona and can only be directly compared with the HF-type II burst. Nevertheless, the model provides an indication of the possible shock region associated with the LF-type II burst.

Figure 12 shows the reconstructed dome of the EIT wave at the start time of the HF-type II burst (23:45 UT on September 27). The EIT wave dome shows the quasi-perpendicular shock normal angle $\left(\theta_{\mathrm{Bn}}\right)$ in the southeast and southwest regions (marked in Fig. 12 by black and green arrows, respectively). 

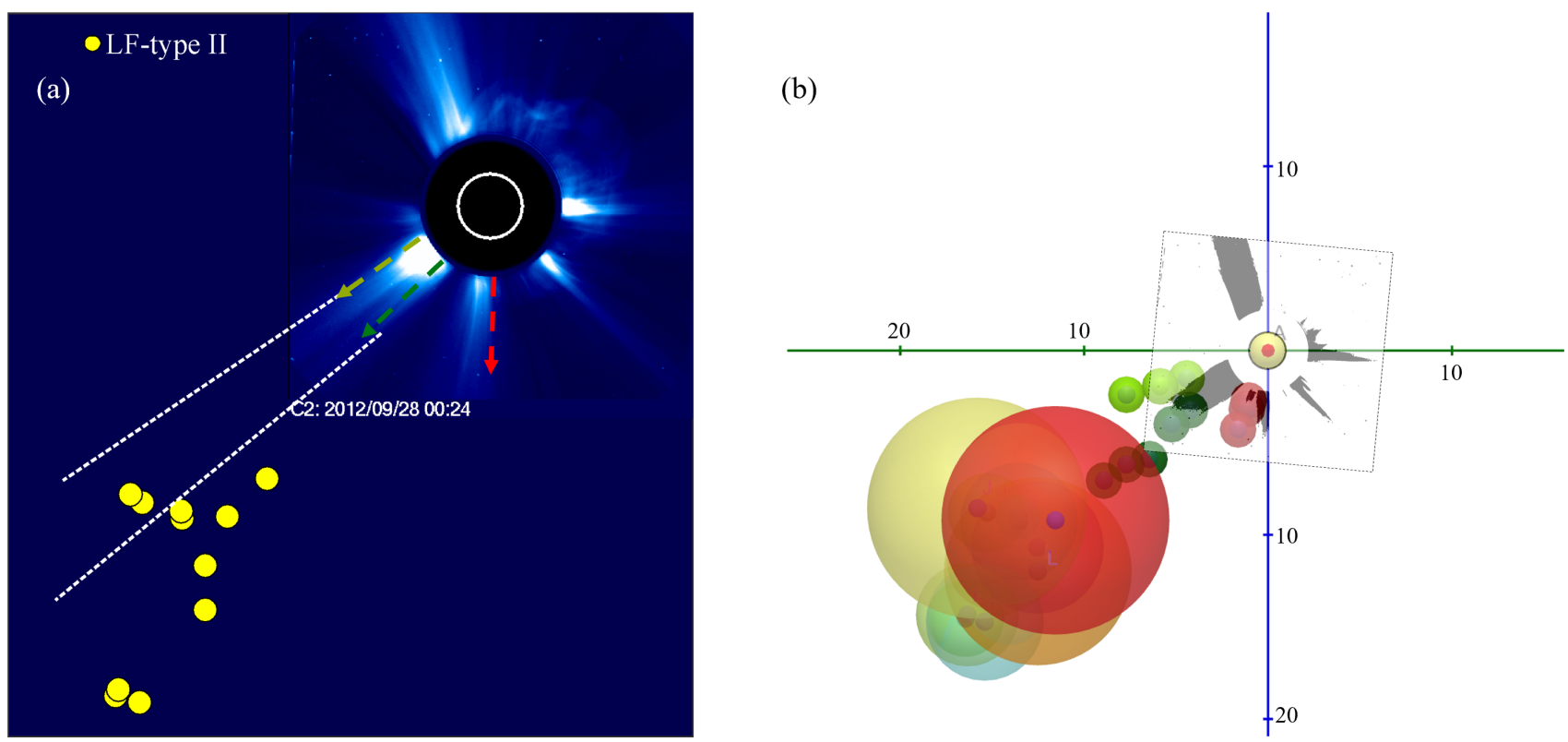

Fig. 11. Panel $a$ : centroids of the LF-type II emission projected on a SOHO/LASCO C2 image. Their proximity to the streamer stalk region in the plane of sky gives an indication of the probable shock/streamer interaction. Coloured arrows are the streamer regions selected for reconstruction. Panel $b$ : reconstructed streamers (colour coded) plotted together with the type II source regions (distance between the wave vectors) in a planeof-sky projection similar to the SOHO/LASCO C2 image. The different frequency pairs of the type II source regions are denoted by a rainbow colour scale (Blue hues: high-frequency pairs, Red hues: low-frequency pairs). The source regions of the type II burst are presented as spheres. The smaller spheres (green, olive, and red) are the positions of the reconstructed streamers.

We believe that the southwest region (green arrow in Fig. 12) is the most probable source location of the HF-type II burst. This conclusion also agrees with the so-called intensity-directivity relationship of the radio emission (Magdalenić et al. 2014). Briefly, the intensity of the HF-type II is strongest as seen by Wind/WAVES, weak as seen by STEREO A/WAVES, and is not observed by STEREO B/WAVES. This suggests that the source of the HF-type II is fully occulted for the STEREO B/WAVES and propagates mostly in the direction of Wind/WAVES.

The southeast region marked by a black arrow in Fig. 12 agrees with the positions of the LF-type II source regions obtained by radio triangulation (at larger heights) and with the fastest CME segments as modelled by EUHFORIA at about $30 R_{\odot}$ (Fig. 3d). Taking all of the above into account, the assumption that the conditions for the quasi-perpendicular regime in the southeast region (as modelled in the low corona, Fig. 12) are also met at larger heights is reasonable. However, if this is not the case, the interaction of the shock wave and streamer can provide an additional favourable condition for the generation of the shock-associated radio emission (as already shown by Shen et al. 2013; Magdalenić et al. 2014; Zucca et al. 2018). Previous studies (e.g. Holman \& Pesses 1983; Mann 1995; Reiner et al. 1998; Mann et al. 2003; Mann \& Klassen 2005, and references therein) have demonstrated that a quasi-perpendicular shock wave geometry is significantly more efficient in accelerating particles and therefore producing radio emission. Some studies have indicated that a shock wave can be radio quiet in the subcritical regime and produce radio emission when supercritical (Gopalswamy et al. 2010, 2012). Nevertheless, the favourable conditions are not only the quasi-perpendicular shock wave geometry but also the high density and the low Alfvén speed in the streamer region, both of which are needed for the generation of type II radio emission (Uchida et al. 1973; Warmuth et al. 2005). We believe that all of the above mentioned conditions, which favour the generation of type II radio bursts, were met in the studied event.

\section{Summary and discussion}

We present a multiwavelength analysis of the CME/flare event on September 27, 2012. The studied C3.7 flare was associated with a full-halo CME (3D speed $\approx 1300 \mathrm{~km} \mathrm{~s}^{-1}$ ), an EIT wave, a coronal dimming, and a WL shock. The speeds of the two type II bursts obtained employing classical method were 1500 and $1000 \mathrm{~km} \mathrm{~s}^{-1}$ for HF and LF type II, respectively. Threedimensional information on the sources of the radio emission was obtained employing the radio triangulation technique and direction finding observations. Radio triangulation revealed the existence of two groups of type III bursts, the flare impulsive (FI) and the flare decay (FD) phase type III bursts. The FI-type IIIs had source regions close to the west CME flank, and FD-type IIIs close to the east CME flank. The LF-type II and FD-type III were found to be roughly co-spatial, appearing in the southeast quadrant of the Sun and close to the eastern flank of the CME. All the studied radio bursts originated from regions of higher density than suggested by the $1 \mathrm{D}$ models. The obtained density profiles crossed several different 1D models. We attributed this behaviour to the strongly non-radial propagation of the radio source.

We found that the EIT wave speed increased from 320 to $770 \mathrm{~km} \mathrm{~s}^{-1}$ when the wave passed through a nearby active region (i.e. the southeast direction from the source region). The modelled EIT wave dome showed a quasi-perpendicular geometry, favourable for the generation of type II radio emission, in two regions of the dome, roughly corresponding to the $\mathrm{CME}$ flanks. Further, we find a good correlation between the position of the LF-type II sources and the nearby streamers. This indicates that the LF-type II radio emission was generated by the shock wave/streamer interaction, which is similar to the findings 

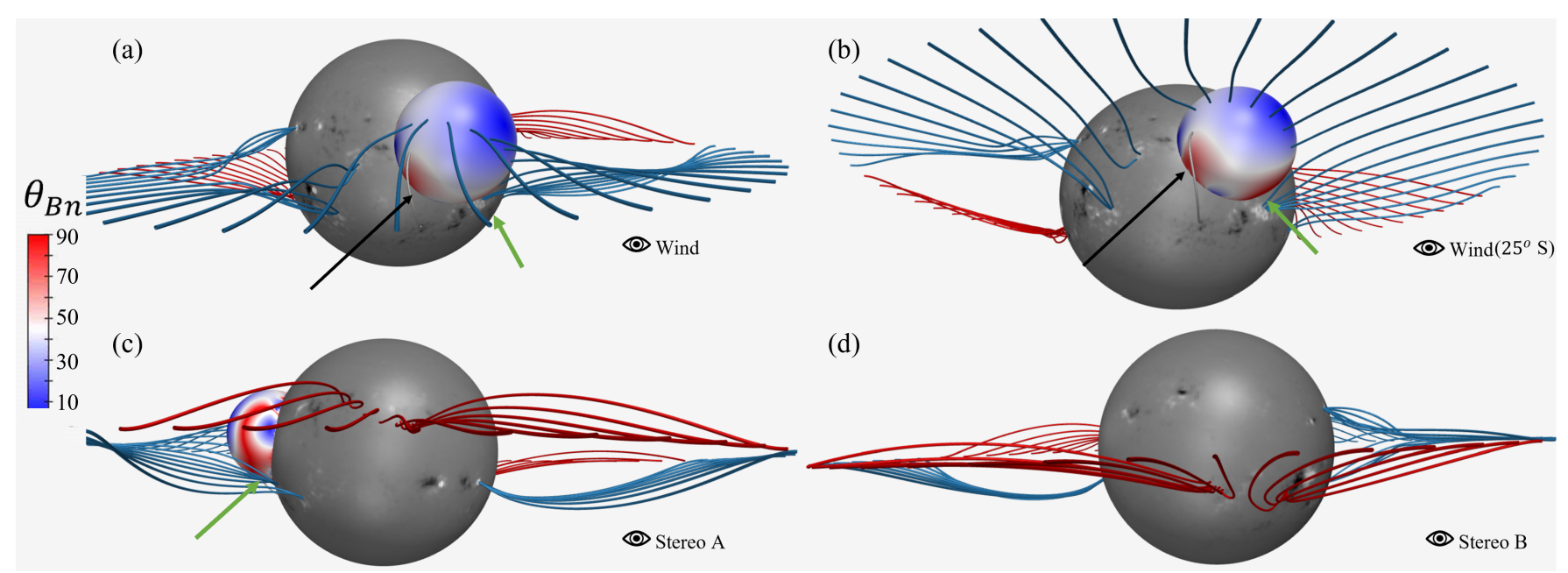

Fig. 12. Reconstructed EIT wave bubble and its corresponding high coronal expansion using the measurements made in the analysis described in Sect. 5. The panels show the event as observed from the point of view of different spacecraft. The different panels show the EIT wave observed $(a)$ by SDO/AIA; $(b)$ from $25^{\circ}$ south; $(c)$ by STEREO A; and $(d)$ by STEREO B. The coronal magnetic field was extrapolated using a PFSS model. The field line colours correspond to their polarity, with red being positive and blue negative. The shock wave bubble is plotted at the start time of the HF-type II (23:44 UT) and the colours on the surface of the bubble are the values of the shock normal angle $\left(\theta_{\mathrm{Bn}}\right)$ to the local magnetic field. The green arrow indicates the possible source region of the HF-type II while the black arrow is the possible region of the part of the shock wave which corresponds to the LF-type II burst.

of studies by Magdalenić et al. (2014), Zucca et al. (2018), and Mancuso et al. (2019). As the HF-type II was observed at a lower frequency than the usual metric-type II bursts (above $150 \mathrm{MHz}$, Klassen et al. 2003; Magdalenić et al. 2010, 2012), it was probably not generated by the flare. We believe that both of the type IIs were driven by the CME. The difference in their starting frequency (and radio source positions) is due to the fact that they are generated in the quasi-perpendicular shock wave regions roughly corresponding to different foot-points of the same CME.

\section{Propagation of the radio emission}

Radio emission can be modified in different ways during its propagation through the corona. Two of the most frequently discussed phenomena are the scattering processes due to local density modulations along the radio emission path (e.g. Fokker 1965; Hollweg 1968; Riddle 1972; Bastian 1994; Arzner \& Magun 1999 ), and the non-radially propagating source of radio emission (e.g. Kundu 1965; Kai 1969; Nelson \& Robinson 1975; Bougeret 1985; Zucca et al. 2018). Due to scattering processes, the position of the radio source might be observed as shifted in comparison with its real position, and its apparent size may be increased (see e.g. Steinberg et al. 1971; Kontar et al. 2017). Different levels of the density fluctuations and their influence on scattering were recently investigated following different approaches (Thejappa et al. 2007; Krupar et al. 2018; Chrysaphi et al. 2018). We note that the majority of these studies consider radial 1D coronal electron density profiles as an input. Radio triangulation is also subject to radio-wave scattering effects, potentially inducing large distances between the wave vectors (Thejappa et al. 2012; Krupar et al. 2016) and accordingly large source regions. As the scattering effects increase with the decrease of the observing frequency, this effect is more pronounced for the lowest direction finding frequencies. Taking this into account, we limited our study to frequency pairs above $500 \mathrm{kHz}$.

Figure 13 presents the radio triangulation results in the ecliptic and meridional plane. The radio source regions correspond to the full distance between the wave vectors (see Sect. 7). The source region diameters of type III bursts at the lowest considered frequencies are about $17 R_{\odot}$. Both, the FI-type III and the FD-type III bursts propagate from the high latitudes towards the ecliptic plan (Fig. 13a). The FI-type IIIs start at the northern hemisphere and FD-type IIIs start at the southern hemisphere; they both show non-radial propagation. Figure 13b shows FI-, FD-, and FD*-type III bursts (green, blue and pink spheres, respectively). We found that the propagation path of the two FDtype IIIs (separated in time by about $5 \mathrm{~min}$ ) is almost identical, with a difference smaller than the apparent sizes of the radio sources. If the scattering effects were found to be significant, we would not expect two type III bursts to have the same propagation path. The solar corona is very dynamic, and scattering of the radio emission due to density fluctuations could induce significantly different radio source positions obtained for the same frequency pairs of these two bursts. As this effect is not observed in the event under study, we believe that the accuracy of the radio triangulation results is within the limits induced by the method itself. Further, the same propagation path of the subsequent type III bursts has already been reported in other studies (Reiner et al. 2009; Klassen et al. 2018; Zhang et al. 2019). We think that the scattering, that can be strongly event dependent (Aurass et al. 1994; Zlotnik et al. 1998), is probably not a dominant process in this event. Even if scattering induces the shift in the source positions to larger heights, it does not significantly affect the propagation direction of the radio emission and our results on the non-radial propagation of the radio emission sources.

Although non-radially propagating radio emission sources are often discussed in the 2D plane (Mann et al. 2003; Carley et al. 2016; Zucca et al. 2018), herein we address this effect in 3D space for the first time. The 3D positions of the LF-type II sources propagating in a strongly non-radial direction are shown in Fig. 14a (different colours represent different frequency pairs).

To demonstrate the effects of the non-radial propagation, we "converted" the 3D positions of the type II sources into two different $1 \mathrm{D}$ radial profiles (Fig. 14). We considered the projection of the $3 \mathrm{D}$ sources to a radial line connecting the centre of the 


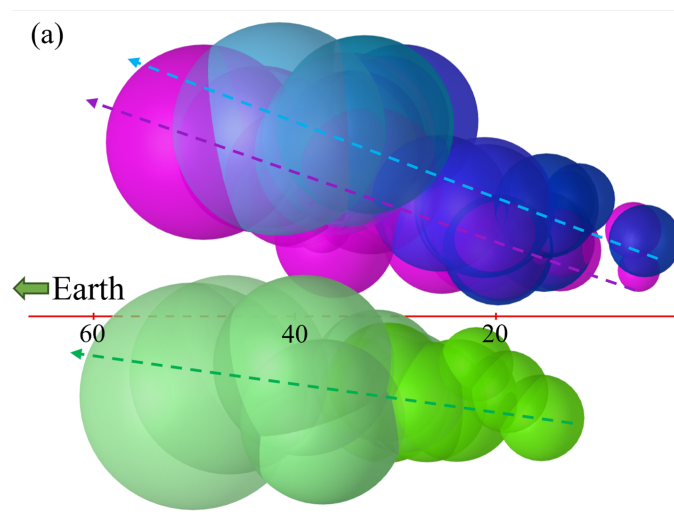

Ecliptic (b)

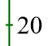

Sun

- - - LF-type II

:- - - - FI-type III

1 $4-----$ FD-type III

20

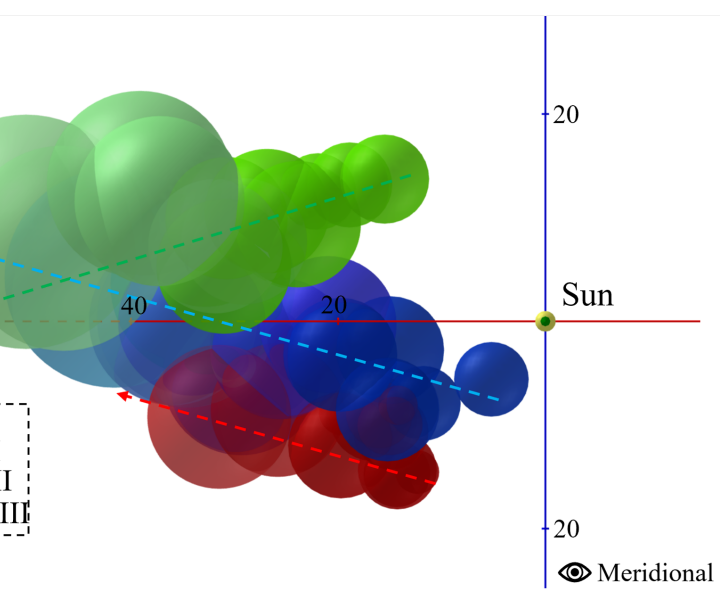

Fig. 13. Radio triangulation results of FI-type III, FD-type III, FD*-type III, and LF-type II plotted together. The units are solar radii $\left(R_{\odot}\right)$ with the Sun at the centre $(0,0,0)$. The dotted lines mark the linear fit to the centroids of the radio sources. Panel $a$ : results in the ecliptic plane. Propagation of all three type III sources is highly non-radial and the two FD-type III bursts seem have a similar propagation path. Panel b: results in the meridional plane. All three bursts show a propagation from high latitude towards the ecliptic plane.

Sun and the radio source of the highest (Fig. 14a), and lowest (Fig. 14b) frequency-pairs (i.e. $925 / 916 \mathrm{kHz}$ and $625 / 624 \mathrm{kHz}$, respectively). The conversion resulted in two $1 \mathrm{D}$ profiles with orthogonal projection of the sources. The right-hand panels of Fig. 14 show how strongly the source region propagation in 1D is different from the non-radial 3D propagation. The obtained 1D profiles also significantly differ from each other because of the differently selected radial directions. The 3D source positions and the projected positions for the same frequency pairs are strongly different depending on the selected radial profile. The distance of the $3 \mathrm{D}$ source position and the projected one can be as large as $13 R_{\odot}$. In the conversion process, the $3 \mathrm{D}$ information was completely lost resulting in erroneous 1D profiles (Fig. 14).

If for the studied event we were to use ground-based interferometric observations, the type II positions would be observed as in Fig. 11b. The strongly non-radial propagation of the radio sources would in this case be observed as almost stationary emission. Taking all this into account, we conclude that employing 1D density profiles in the study of propagation of the radio emission needs to be done whilst taking into account the possibility of large errors, and could be considered only as a very rough approximation. Further, estimation of the level of scattering effects (Kontar et al. 2017; Chrysaphi et al. 2018; McCauley et al. 2018) should also take into account the possible influence of the non-radial propagation and the projection effects. Gordovskyy et al. (2019) employed different corrections for projection effects and obtained significant changes in the estimated source heights. However, due to the lack of spatial information, the results were attributed to the scattering effects. It is probable that drawing general conclusions is difficult, as both density fluctuations and the propagation direction of radio emission might strongly change from event to event.

\section{Conclusions}

The relationship between CME/flare events, shocks, and associated type II radio bursts has been extensively discussed for several decades (e.g. Cairns et al. 2003, and references therein). This study brings some new and important findings on the association of the radio emission and solar eruptive phenomena. Here, we list the most important results:

- Radio triangulation studies of type III bursts have been performed in the past, but we show for the first time that the source positions of type III bursts observed during a single eruptive event were located in significantly different locations. We find that the FI-type III bursts (observed during flare impulsive phase) originate from close to the western CME-flank region, and the FD-type III bursts (observed during the flare decay phase) originate from close to the eastern CME-flank region.

- We find the propagation path of two subsequent type III bursts are very similar (FD- and FD*-type III, Fig. 13a), with differences smaller than the source region sizes (i.e. distance between two wave vectors). We do not find any significant difference in the source positions for the same frequency pairs for these two bursts, which would be expected if the scattering processes in this event were significant. The accuracy of the radio triangulation is therefore within the limits induced by the radio triangulation method.

- One of the two type II bursts (HF- and LF-type II) associated with the studied event, the LF-type II, starts at an unusually low frequency. We find that the LF-type II was associated with the interaction of the shock wave and a streamer region. Although appearing at very different parts of the CME (different flanks), both of the type II radio bursts seem to be driven by the CME.

- The radio triangulation study of the LF-type II burst provides evidence of the strongly non-radial propagation of the radio sources. Although this has already been discussed previously (Kai 1969; Bougeret 1985, and references therein), only the 3D information obtained in the radio triangulation allows us to quantify the effects associated with the non-radial propagation.

- The coronal electron densities obtained in the present radio triangulation study show that all radio bursts in this event are generated in the regions of higher density than what is usually considered when employing 1D density models. This is to be expected in particular during periods of high solar activity and at times when the global magnetic field of the Sun is very complex. Therefore, employing the 1D density models for explaining radio emission should be considered with great care, and only as a first level approximation.

- The EIT wave associated with the eruptive event accelerates (from 320 to $770 \mathrm{~km} \mathrm{~s}^{-1}$ ) when passing a nearby active region, in the direction roughly coinciding with the propagation direction of the LF-type II. The reconstructed dome 

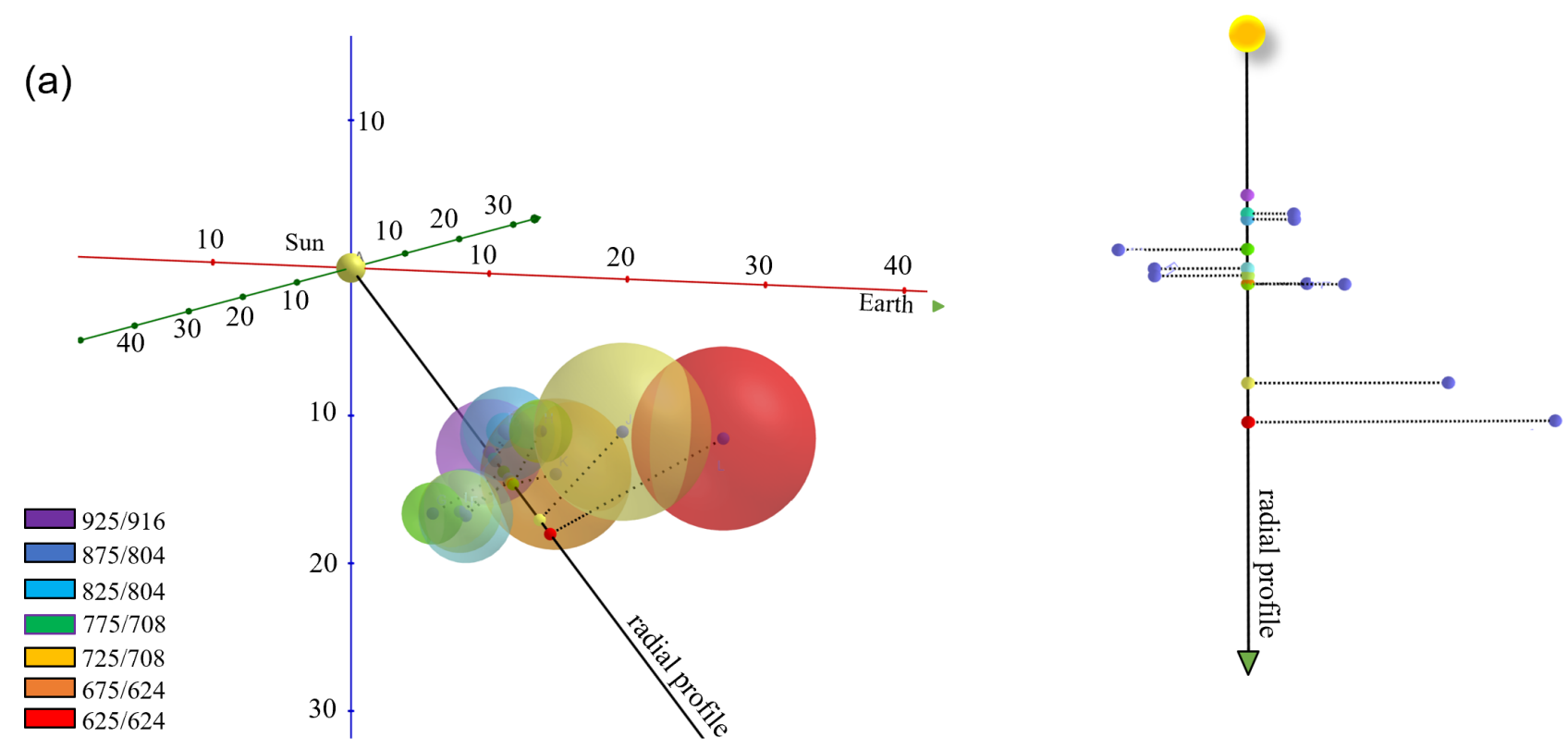

(b)
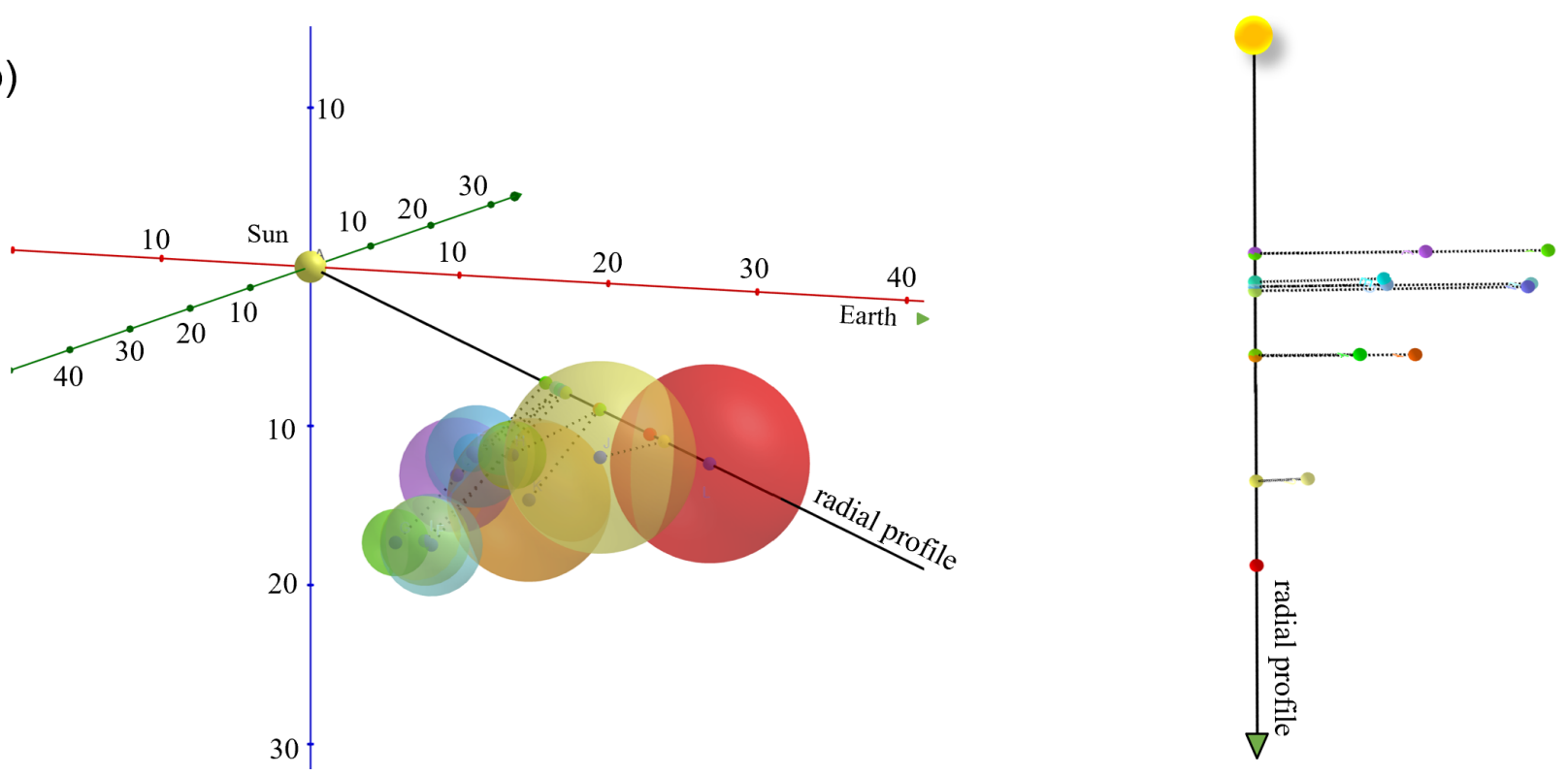

Fig. 14. Radio triangulation results of LF-type II plotted in detail to show the effect of non-radial propagation of the emitting source. The units are solar radii $\left(R_{\odot}\right)$, and the Sun is at the center of the coordinate system. A ray is drawn from the centre of the Sun $(0,0,0)$ or $R_{\odot}=0$ to centres of different sources. Panel $a$ : result when the ray is drawn employing the highest frequency (Purple, 925/916 kHz) pair. The projection is shown in detail in the line profile adjacent to it. Panel $b$ : result when the ray is drawn towards the lowest frequency (Red, 625/624 kHz) pair. The result of the orthogonal projection is shown in the profile adjacent to it.

of the EIT wave indicates the existence of two main regions with quasi-perpendicular shock regimes, roughly associated with the CME-flanks. The southwest region is most probably the source region of the HF-type II burst, and the southeast region of the quasi-perpendicular geometry is the source region of the LF-type II burst.

Radio triangulation is not dependent on a density model and therefore provides a unique opportunity to study different aspects of the radio bursts and their association with the solar transients. The radio emission can be influenced in different ways during its propagation through the corona, and this will also affect the results of the radio triangulation. Therefore, as with all other observations, gonipolarimetric observations need to be treated with care, and their limitations must be taren into account. Nevertheless, direction finding observations provide unique informa- tion on the 3D positions of the radio emission, and can help us to understand the processes of radio emission during eruptive events in an unprecedented way.

Acknowledgements. EIT and LASCO data have been used courtesy of the SOHO/EIT and SOHO/LASCO consortiums, respectively. The STEREO SECCHI data are produced by a consortium of RAL(UK), NRL(USA), LMSAL(USA), GSFC(USA), MPS(Germany), CSL(Belgium), IOTA(France), and IAS(France). The Wind/Waves instrument was designed and built as a joint effort of the Paris-Meudon Observatory, the University of Minnesota, and the Goddard Space Flight Center, and the data are available at the instrument Web site. We thank the radio monitoring service at LESIA (Observatoire de Paris) for providing value-added data that have been used for this study. We are grateful to the staff of the Bruny Island Radio Spectrometer for their open data policy. The authors are grateful for useful discussions with Dr. Bojan Vršnak, Dr. Eduard Kontar, and Dr. Milan Maksimovic, regarding the propagation of the radio emission and scattering effects. The authors are also thankful to the anonymous referee for their valuable input which helped us to significantly 
improve the manuscript. I.C.J. was supported by a $\mathrm{PhD}$ grant awarded by the Royal Observatory of Belgium. C.S. acknowledges funding from the Research Foundation - Flanders (FWO, fellowship no. 1S42817N). K.D. and A.M.V. acknowledge funding by the Austrian Space Applications Programme of the Austrian Research Promotion Agency FFG (ASAP-11 4900217 BMVIT) and the Austrian Science Fund FWF: P24092-N16 and P27292-N20. EK acknowledges Finnish Centre of Excellence in Research of Sustainable Space (Academy of Finland grant number 1312390), European Research Council (ERC) unde the European Union's Horizon 2020 research and innovation programme (ERC COG 724391), and Academy of Finland project SMASH no. 310445. EUHFORIA is developed as a joint effort between the University of Helsinki and KU Leuven. The validation of solar wind and CME modelling with EUHFORIA is being performed within the BRAIN-be project CCSOM (Constraining CMEs and Shocks by Observations and Modelling throughout the inner heliosphere; www.sidc.be/ccsom/) and BRAIN-be project SWiM (Solar Wind Modeling with EUHFORIA for the new heliospheric missions).

\section{References}

Arzner, K., \& Magun, A. 1999, A\&A, 351, 1165

Aschwanden, M. J. 2019, New Millennium Solar Physics (Switzerland, AG: Springer Nature), 458

Aurass, H., Klein, K. L., \& Mann, G. 1994, in Solar Dynamic Phenomena an Solar Wind Consequences, the Third SOHO Workshop, ed. J. J. Hunt, ESA Spec. Publ., 373, 95

Bastian, T. S. 1994, ApJ, 426, 774

Benz, A. O. 2017, Liv. Rev. Sol. Phys., 14, 2

Bougeret, J. L. 1985, Geophysical Monograph Series (Washington, DC: American Geophysical Union), 35, 13

Bougeret, J.-L., Kaiser, M. L., Kellogg, P. J., et al. 1995, Space Sci. Rev., 71, 231 Bougeret, J. L., Goetz, K., Kaiser, M. L., et al. 2008, Space Sci. Rev., 136, 487

Brueckner, G. E., Howard, R. A., Koomen, M. J., et al. 1995, Sol. Phys., 162 357

Cairns, I. H., Knock, S. A., Robinson, P. A., \& Kuncic, Z. 2003, Space Sci. Rev. 107,27

Carley, E. P., Vilmer, N., \& Gallagher, P. T. 2016, ApJ, 833, 87

Cecconi, B., \& Zarka, P. 2005, Radio Sci., 40, RS3003

Cecconi, B., Bonnin, X., Hoang, S., et al. 2008, Space Sci. Rev., 136, 549

Chrysaphi, N., Kontar, E. P., Holman, G. D., \& Temmer, M. 2018, ApJ, 868 , 79

Cremades, H., St. Cyr, O. C., \& Kaiser, M. L. 2007, Space Weather, 5, 8001

Dissauer, K., Veronig, A. M., Temmer, M., Podladchikova, T., \& Vanninathan, K. 2018, ApJ, 863, 169

Dissauer, K., Veronig, A. M., Temmer, M., \& Podladchikova, T. 2019, ApJ, 874, 123

Domingo, V., Fleck, B., \& Poland, A. I. 1995, Sol. Phys., 162, 1

Erickson, W. C. 1997, PASA, 14, 278

Eselevich, V. G., Eselevich, M. V., \& Zimovets, I. V. 2019, Sol. Phys., 294, 73

Fainberg, J., \& Stone, R. G. 1974, Space Sci. Rev., 16, 145

Fainberg, J., Evans, L. G., \& Stone, R. G. 1972, Science, 178, 743

Feng, S. W., Chen, Y., Kong, X. L., et al. 2012, ApJ, 753, 21

Fletcher, L., Dennis, B. R., Hudson, H. S., et al. 2011, Space Sci. Rev., 159, 19

Floyd, O., Lamy, P., \& Llebaria, A. 2014, Sol. Phys., 289, 1313

Fokker, A. D. 1965, Bull. Astron. Inst. Neth., 18, 111

Garcia, H. A. 1994, Sol. Phys., 154, 275

Gary, D. E., Dulk, G. A., House, L., et al. 1984, A\&A, 134, 222

Ginzburg, V. L., \& Zhelezniakov, V. V. 1958, Sov. Astron., 2, 653

Gopalswamy, N., Xie, H., Mäkelä, P., et al. 2010, ApJ, 710, 1111

Gopalswamy, N., MäKelä, P., Akiyama, S., et al. 2012, J. Geophys. Res. (Space Phys.), 117, A08106

Gordovskyy, M., Kontar, E., Browning, P., \& Kuznetsov, A. 2019, ApJ, 873, 48

Green, L. M., Török, T., Vršnak, B., Manchester, W., \& Veronig, A. 2018, Space Sci. Rev., 214, 46

Gurnett, D. A., Baumback, M. M., \& Rosenbauer, H. 1978, J. Geophys. Res., 83 616

Hinterreiter, J., Magdalenic, J., Temmer, M., et al. 2019, Sol. Phys., 294, 170

Hoang, S., Maksimovic, M., Bougeret, J.-L., Reiner, M. J., \& Kaiser, M. L. 1998, Geophys. Rev. Lett., 25, 2497

Hollweg, J. V. 1968, AJ, 73, 972

Holman, G. D., \& Pesses, M. E. 1983, ApJ, 267, 837

Howard, R. A., Moses, J. D., Vourlidas, A., et al. 2008, Space Sci. Rev., 136, 67 Hudson, H. S., Acton, L. W., \& Freeland, S. L. 1996, ApJ, 470, 629

Inhester, B. 2006, ArXiv e-prints [arXiv:astro-ph/0612649]

Kai, K. 1969, Sol. Phys., 10, 460

Kaiser, M. L. 2005, Adv. Space Res., 36, 1483

Kaiser, M. L., Kucera, T. A., Davila, J. M., et al. 2008, Space Sci. Rev., 136, 5

Kienreich, I. W., Temmer, M., \& Veronig, A. M. 2009, ApJ, 703, L118
Klassen, A., Aurass, H., Klein, K., Hofmann, A., \& Mann, G. 1999, A\&A, 343 287

Klassen, A., Pohjolainen, S., \& Klein, K. 2003, Sol. Phys., 218, 197

Klassen, A., Dresing, N., Gómez-Herrero, R., Heber, B., \& Veronig, A. 2018, A\&A, 614, A61

Klein, K.-L., Khan, J. I., Vilmer, N., Delouis, J.-M., \& Aurass, H. 1999, A\&A, 346, L53

Kontar, E. P., Yu, S., Kuznetsov, A. A., et al. 2017, Nat. Commun., 8, 1515

Kontar, E. P., Chen, X., Chrysaphi, N., et al. 2019, ApJ, 884, 122

Krupar, V., Santolik, O., Cecconi, B., et al. 2012, J. Geophys. Res. (Space Phys.), 117,6101

Krupar, V., Kontar, E. P., Soucek, J., et al. 2015, A\&A, 580, A137

Krupar, V., Eastwood, J. P., Kruparova, O., et al. 2016, ApJ, 823, L5

Krupar, V., Maksimovic, M., Kontar, E. P., et al. 2018, ApJ, 857, 82

Krupar, V., Magdalenić, J., Eastwood, J. P., et al. 2019, ApJ, 882, 92

Krupar, V., Szabo, A., Maksimovic, M., et al. 2020, ApJS, 246, 57

Kumar, P., Innes, D. E., \& Cho, K.-S. 2016, ApJ, 828, 28

Kundu, M. R. 1965, Solar Radio Astronomy (New York: Interscience Publication)

Leblanc, Y., Dulk, G. A., \& Bougeret, J.-L. 1998, Sol. Phys., 183, 165

Lecacheux, A. 1978, A\&A, 70, 701

Lemen, J. R., Title, A. M., Akin, D. J., et al. 2012, Sol. Phys., 275, 17

Liu, Y. D., Luhmann, J. G., Kajdič, P., et al. 2014, Nat. Commun., 5, 3481

Long, D. M., Bloomfield, D. S., Chen, P. F., et al. 2017, Sol. Phys., 292, 7

Magdalenić, J., Vršnak, B., Pohjolainen, S., et al. 2008, Sol. Phys., 253, 305

Magdalenić, J., Marqué, C., Zhukov, A. N., Vršnak, B., \& Žic, T. 2010, ApJ, 718, 266

Magdalenić, J., Marqué, C., Zhukov, A. N., Vršnak, B., \& Veronig, A. 2012, ApJ, 746, 152

Magdalenić, J., Marqué, C., Krupar, V., et al. 2014, ApJ, 791, 115

Maia, D., Pick, M., Vourlidas, A., \& Howard, R. 2000, ApJ, 528, L49

Mäkelä, P., Gopalswamy, N., Reiner, M. J., Akiyama, S., \& Krupar, V. 2016, ApJ, 827, 141

Mäkelä, P., Gopalswamy, N., \& Akiyama, S. 2018, ApJ, 867, 40

Mancuso, S., Frassati, F., Bemporad, A., \& Barghini, D. 2019, A\&A, 624, L2

Mann, G. 1995, in Theory and Observations of Coronal Shock Waves, eds. A. O. Benz, \& A. Krüger, 444, 183

Mann, G., \& Klassen, A. 2005, A\&A, 441, 319

Mann, G., Jansen, F., MacDowall, R. J., Kaiser, M. L., \& Stone, R. G. 1999a, A\&A, 348, 614

Mann, G., Aurass, H., Klassen, A., Estel, C., \& Thompson, B. J. 1999b, in 8th SOHO Workshop: Plasma Dynamics and Diagnostics in the Solar Transition Region and Corona, eds. J. C. Vial, \& B. Kaldeich-Schü, ESA Spec. Publ., 446, 477

Mann, G., Klassen, A., Aurass, H., \& Classen, H. T. 2003, A\&A, 400, 329

Manning, R., \& Fainberg, J. 1980, Space Sci. Instrum., 5, 161

Martínez-Oliveros, J. C., Raftery, C. L., Bain, H. M., et al. 2012a, ApJ, 748, 66

Martínez-Oliveros, J. C., Lindsey, C., Bale, S. D., \& Krucker, S. 2012b, Sol. Phys., 279, 153

Martínez-Oliveros, J. C., Raftery, C., Bain, H., et al. 2015, Sol. Phys., 290, 891

McCauley, P. I., Cairns, I. H., \& Morgan, J. 2018, Sol. Phys., 293, 132

Melrose, D. B. 1970, Aust. J. Phys., 23, 871

Melrose, D. B. 1980, Space Sci. Rev., 26, 3

Melrose, D. B. 2017, Rev. Mod. Plasma Phys., 1, 5

Miteva, R., Samwel, S. W., \& Krupar, V. 2017, J. Space Weather Space Clim., 7, A37

Muhr, N., Vršnak, B., Temmer, M., Veronig, A. M., \& Magdalenić, J. 2010, ApJ, 708, 1639

Nelson, G. J., \& Robinson, R. D. 1975, PASA, 2, 370

Nindos, A., Alissandrakis, C. E., Hillaris, A., \& Preka-Papadema, P. 2011, A\&A, 531, A31

Odstrčil, D., \& Pizzo, V. J. 1999, J. Geophys. Res., 104, 483

Odstrčil, D., Dryer, M., \& Smith, Z. 1996, J. Geophys. Res., 101, 19973

Palmerio, E., Scolini, C., Barnes, D., et al. 2019, ApJ, 878, 37

Patsourakos, S., \& Vourlidas, A. 2009, ApJ, 700, L182

Pesnell, W. D., Thompson, B. J., \& Chamberlin, P. C. 2012, Sol. Phys., 275, 3

Podladchikova, O., \& Berghmans, D. 2005, Sol. Phys., 228, 265

Podladchikova, T., Van der Linden, R., \& Veronig, A. M. 2017, ApJ, 850, 81

Podladchikova, T., Veronig, A. M., Dissauer, K., Temmer, M., \& Podladchikova, O. 2019, ApJ, 877, 68

Pohjolainen, S. 2008, A\&A, 483, 297

Pomoell, J., \& Poedts, S. 2018, J. Space Weather Space Clim., 8, A35

Reid, H. A. S., \& Ratcliffe, H. 2014, Res. Astron. Astrophys., 14, 773

Reid, H. A. S., Vilmer, N., \& Kontar, E. P. 2014, A\&A, 567, A85

Reiner, M. J., \& Stone, R. G. 1988, A\&A, 206, 316

Reiner, M. J., Fainberg, J., Kaiser, M. L., \& Stone, R. G. 1998, J. Geophys. Res., 103,1923 
Reiner, M. J., Kaiser, M. L., Gopalswamy, N., et al. 2001, J. Geophys. Res., 106, 25279

Reiner, M. J., Goetz, K., Fainberg, J., et al. 2009, Sol. Phys., 259, 255

Riddle, A. C. 1972, PASA, 2, 148

Rouillard, A. P., Plotnikov, I., Pinto, R. F., et al. 2016, ApJ, 833, 45

Saito, K. 1970, Annal. Tokyo Astron. Obs., 12, 51

Santolík, O., Parrot, M., \& Lefeuvre, F. 2012, Radio Sci., 38, 1010

Schrijver, C. J., \& De Rosa, M. L. 2003, Sol. Phys., 212, 165

Scolini, C., Verbeke, C., Poedts, S., et al. 2018, Space Weather, 16, 754

Scolini, C., Rodriguez, L., Mierla, M., Pomoell, J., \& Poedts, S. 2019, A\&A, 626, A122

Scolini, C., Chané, E., Temmer, M., et al. 2020, ApJS, 247, 21

Shen, C., Liao, C., Wang, Y., Ye, P., \& Wang, S. 2013, Sol. Phys., 282, 543

Shibata, K., \& Magara, T. 2011, Liv. Rev. Sol. Phys., 8, 6

Steinberg, J. L., Aubier-Giraud, M., Leblanc, Y., \& Boischot, A. 1971, A\&A, 10, 362

Temmer, M., \& Nitta, N. V. 2015, Sol. Phys., 290, 919

Temmer, M., Veronig, A. M., Gopalswamy, N., \& Yashiro, S. 2011, Sol. Phys., 273,421

Thejappa, G., MacDowall, R. J., \& Kaiser, M. L. 2007, ApJ, 671, 894

Thejappa, G., MacDowall, R. J., \& Bergamo, M. 2012, ApJ, 745, 187

Thernisien, A. F. R., Howard, R. A., \& Vourlidas, A. 2006, ApJ, 652, 763

Thernisien, A., Vourlidas, A., \& Howard, R. A. 2009, Sol. Phys., 256, 111

Thompson, B. J., Plunkett, S. P., Gurman, J. B., et al. 1998, Geophys. Rev. Lett., 25,2465
Uchida, Y., Altschuler, M. D., Newkirk, G., Jr. 1973, Sol. Phys., 28, 495

Veronig, A. M., Temmer, M., Vršnak, B., \& Thalmann, J. K. 2006, ApJ, 647, 1466

Veronig, A. M., Temmer, M., \& Vršnak, B. 2008, ApJ, 681, L113

Veronig, A. M., Muhr, N., Kienreich, I. W., Temmer, M., \& Vršnak, B. 2010, ApJ, 716, L57

Veronig, A. M., Podladchikova, T., Dissauer, K., et al. 2018, ApJ, 868, 107

Veronig, A. M., Gömöry, P., Dissauer, K., Temmer, M., \& Vanninathan, K. 2019, ApJ, 879, 85

Vršnak, B., \& Cliver, E. W. 2008, Sol. Phys., 253, 215

Vršnak, B., Warmuth, A., Temmer, M., et al. 2006, A\&A, 448, 739

Warmuth, A. 2015, Liv. Rev. Sol. Phys., 12, 3

Warmuth, A., Vršnak, B., Magdalenić, J., Hanslmeier, A., \& Otruba, W. 2004, A\&A, 418, 1101

Warmuth, A., Mann, G., \& Aurass, H. 2005, ApJ, 626, L121

Wild, J. P. 1950, Aust. J. Sci. Res. A Phys. Sci., 3, 399

Wild, J. P., \& McCready, L. L. 1950, Aust. J. Sci. Res. A Phys. Sci., 3, 387

Zhang, P., Wang, C., Ye, L., \& Wang, Y. 2019, Sol. Phys., 294, 62

Zhukov, A. N., \& Auchère, F. 2004, A\&A, 427, 705

Zimovets, I., Vilmer, N., Chian, A. C.-L., Sharykin, I., \& Struminsky, A. 2012, A\&A, 547, A6

Zlotnik, E. Y., Klassen, A., Klein, K. L., Aurass, H., \& Mann, G. 1998, A\&A, 331, 1087

Zucca, P., Carley, E. P., Bloomfield, D. S., \& Gallagher, P. T. 2014, A\&A, 564, A47

Zucca, P., Morosan, D. E., Rouillard, A. P., et al. 2018, A\&A, 615, A89 